AperTO - Archivio Istituzionale Open Access dell'Università di Torino

\title{
A nonlocal supercritical Neumann problem
}

\section{This is the author's manuscript}

Original Citation:

Availability:

This version is available http://hdl.handle.net/2318/1713459

since 2020-01-03T13:04:22Z

Published version:

DOI:10.1016/j.jde.2019.09.014

Terms of use:

Open Access

Anyone can freely access the full text of works made available as "Open Access". Works made available under a Creative Commons license can be used according to the terms and conditions of said license. Use of all other works requires consent of the right holder (author or publisher) if not exempted from copyright protection by the applicable law. 


\section{A nonlocal supercritical Neumann problem}

\section{Eleonora Cinti $^{\mathrm{a}}$, Francesca Colasuonno ${ }^{\mathrm{b}}$}

a Dipartimento di Matematica, Alma Mater Studiorum Università di Bologna, piazza di Porta San Donato, 5, 40126 Bologna, Italy

b Dipartimento di Matematica “Giuseppe Peano", Università degli Studi di Torino, via Carlo Alberto, 10, 10123 Torino, Italy

Received 4 April 2019; accepted 10 September 2019

We establish existence of positive non-decreasing radial solutions for a nonlocal nonlinear Neumann problem both in the ball and in the annulus. The nonlinearity that we consider is rather general, allowing for supercritical growth (in the sense of Sobolev embedding). The consequent lack of compactness can be overcome, by working in the cone of non-negative and non-decreasing radial functions. Within this cone, we establish some a priori estimates which allow, via a truncation argument, to use variational methods for proving existence of solutions. As a side result, we prove a strong maximum principle for nonlocal Neumann problems, which is of independent interest.

(c) 2019 Elsevier Inc. All rights reserved.

MSC: 35R11; 35B09; 35B 45; 35A15; 60G22

Keywords: Nonlocal elliptic equation; Fractional Laplacian; Sobolev-supercritical nonlinearities; Nonlocal Neumann boundary conditions; Variational methods

E-mail addresses: eleonora.cinti5@unibo.it (E. Cinti), francesca.colasuonno@unito.it (F. Colasuonno). 


\section{Introduction}

For $s>1 / 2$, we consider the following nonlocal Neumann problem

$$
\begin{cases}(-\Delta)^{s} u+u=f(u) & \text { in } \Omega, \\ u \geq 0 & \text { in } \Omega, \\ \mathcal{N}_{s} u=0 & \text { in } \mathbb{R}^{n} \backslash \bar{\Omega} .\end{cases}
$$

Here $\Omega$ is a radial domain of $\mathbb{R}^{n}$, it is either a ball

$$
\Omega=B_{R}:=\left\{x \in \mathbb{R}^{n}:|x|<R\right\}, \quad R>0,
$$

or an annulus

$$
\Omega=A_{R_{0}, R}:=\left\{x \in \mathbb{R}^{n}: R_{0}<|x|<R\right\}, \quad 0<R_{0}<R .
$$

Furthermore, $n \geq 1,(-\Delta)^{s}$ denotes the fractional Laplacian

$$
(-\Delta)^{s} u(x):=c_{n, s} \mathrm{PV} \int_{\mathbb{R}^{n}} \frac{u(x)-u(y)}{|x-y|^{n+2 s}} d y,
$$

and $\mathcal{N}_{s}$ is the following nonlocal normal derivative

$$
\mathcal{N}_{s} u(x):=c_{n, s} \int_{\Omega} \frac{u(x)-u(y)}{|x-y|^{n+2 s}} d y \quad \text { for all } x \in \mathbb{R}^{n} \backslash \bar{\Omega}
$$

first introduced in [11], and $c_{n, s}$ is a normalization constant. It is a well known fact that the fractional Laplacian $(-\Delta)^{s}$ is the infinitesimal generator of a Lévy process. The notion of nonlocal normal derivative $\mathcal{N}_{S}$ has also a particular probabilistic interpretation; we will comment on it later on in Section 2. We stress here that, with this definition of nonlocal Neumann boundary conditions, problem (1.1) has a variational structure.

In this paper, we study the existence of non-constant solutions of (1.1) for a superlinear nonlinearity $f$, which can possibly be supercritical in the sense of Sobolev embeddings.

In order to state our main result, we introduce the hypotheses on $f$. We assume that $f \in$ $C^{1, \gamma}([0, \infty))$, for some $\gamma>0$, satisfies the following conditions:

$\left(f_{1}\right) f^{\prime}(0)=\lim _{t \rightarrow 0^{+}} \frac{f(t)}{t} \in(-\infty, 1)$;

$\left(f_{2}\right) \liminf _{t \rightarrow \infty} \frac{f(t)}{t}>1$;

$\left(f_{3}\right)$ there exists a constant $u_{0}>0$ such that $f\left(u_{0}\right)=u_{0}$ and $f^{\prime}\left(u_{0}\right)>\lambda_{2}^{+, \mathrm{r}}+1$,

where $\lambda_{2}^{+, \mathrm{r}}>0$ is the second radial increasing eigenvalue of the fractional Laplacian with (nonlocal) Neumann boundary conditions.

Clearly, as a consequence of $\left(f_{1}\right)$, we know that $f(0)=0$ and $f$ is below the line $t$ in a right neighborhood of 0 . The results of the paper continue to hold if we weaken $\left(f_{1}\right)$ as follows 
$\left(f_{1}^{\prime}\right) f(0)=0, f^{\prime}(0) \in(-\infty, 1]$ and $f(t)<t$ in $(0, \bar{t})$ for some $\bar{t}>0$.

A prototype nonlinearity satisfying $\left(f_{1}\right)$ and $\left(f_{2}\right)$ is given by

$$
f(t):=t^{q-1}-t^{r-1} \text {, with } 2 \leq r<q .
$$

For $q$ large enough, the above function satisfies condition $\left(f_{3}\right)$ as well.

We observe that $\left(f_{1}\right)$ and $\left(f_{2}\right)$ are enough to prove the existence of a mountain pass-type solution. The additional hypothesis $\left(f_{3}\right)$ is needed to prove that such a solution is non-constant. In particular, the existence of a fixed point $u_{0}$ of $f$ is a consequence of $\left(f_{1}\right),\left(f_{2}\right)$, and the regularity of $f$; moreover, in view of $\int_{\Omega}(-\Delta)^{s} u d x=0$ (cf. (2.3) below), the fact that $f(t)-t$ must change sign at least once is a natural compatibility condition for the existence of solutions.

Our main result can be stated as follows.

Theorem 1.1. Let $s>1 / 2$ and $f \in C^{1, \gamma}([0, \infty))$, for some $\gamma>0$, satisfy assumptions $\left(f_{1}\right)-\left(f_{3}\right)$. Then there exists a non-constant, radial, radially non-decreasing solution of (1.1) which is of class $C^{2}$ and positive almost everywhere in $\Omega$. In addition, if $u_{0,1}, \ldots, u_{0, N}$ are $N$ different positive constants satisfying $\left(f_{3}\right)$, then (1.1) admits $N$ different non-constant, radial, radially non-decreasing, a.e. positive solutions.

If $\Omega=A_{R_{0}, R}$, the same existence and multiplicity result holds also for non-constant, radial, radially non-increasing, a.e. positive $C^{2}$ solutions of (1.1).

We stress here that the situation with Neumann boundary conditions is completely different from the case with Dirichlet boundary conditions. Indeed, as for the local case $s=1$, a Pohožaevtype identity implies nonexistence of solutions under Dirichlet boundary conditions for critical or supercritical nonlinearities, cf. [13, Corollary 1.3], while here, under Neumann boundary conditions, we can find solutions even in the supercritical regime. Moreover, the supercritical nature of the problem prevents a priori the use of variational methods to attack the problem. Indeed, the energy functional associated to (1.1) is not even well-defined in the natural space where we look for solutions, i.e., $H_{\Omega, 0}^{s}$ (cf. Section 3). To overcome this issue, we follow essentially the strategy used in $[4,8]$. Our starting point is to work in the cone of non-negative, radial, non-decreasing functions

$$
\mathcal{C}_{+}(\Omega):=\left\{u \in H_{\Omega, 0}^{s}: \begin{array}{l}
u \text { is radial and } u \geq 0 \text { in } \mathbb{R}^{n}, \\
u(r) \leq u(s) \text { for all } R_{0} \leq r \leq s \leq R
\end{array}\right\}
$$

where with abuse of notation we write $u(|x|):=u(x)$ and in order to treat simultaneously the two cases $\Omega=B_{R}$ and $\Omega=A_{R_{0}, R}$, we assimilate $B_{R}$ into the limit case $A_{0, R}$. This cone was introduced for the local case $(s=1)$ by Serra and Tilli in [14], it is convex and closed in the $H^{s}$-topology. The idea of working with radial functions, suggested by the symmetry of the problem, is dictated by the necessity of gaining compactness. Indeed, restricting the problem to the space of radial $H^{s}$ functions $\left(H_{\text {rad }}^{s}\right)$ allows somehow to work in a 1-dimensional domain, where we have better embeddings than in higher dimension. Nevertheless, in the case of the ball, the energy functional is not well defined even in $H_{\text {rad }}^{s}$, since the sole radial symmetry is not enough to prevent the existence of sequences of solutions exploding at the origin. This is the reason for the increasing monotonicity request in the cone $\mathcal{C}_{+}$, cf. [9] for similar arguments in more general 
domains. Indeed, we can prove that all solutions of (1.1) belonging to $\mathcal{C}_{+}$are a priori bounded in $H_{\Omega, 0}^{s}$ and in $L^{\infty}(\Omega)$. When the domain does not contain the origin, i.e. in the case of the annulus $R_{0}>0$, the monotonicity request can be avoided and it is possible to work directly in the space $H_{\text {rad }}^{s}$. Nonetheless, working in $H_{\text {rad }}^{s}$ would allow to prove the existence of just one radial weak solution of the equation in (1.1) under Neumann boundary conditions, whose sign and monotonicity are not known. Therefore, also in the case of the annulus, even if we do not need to gain compactness, we will work in $\mathcal{C}_{+}\left(A_{R_{0}, R}\right)$ to find a non-decreasing solution, and in

$$
\mathcal{C}_{-}\left(A_{R_{0}, R}\right):=\left\{u \in H_{A_{R_{0}, R}, 0}^{s}: \begin{array}{l}
u \text { is radial and } u \geq 0 \text { in } \mathbb{R}^{n}, \\
u(r) \geq u(s) \text { for all } R_{0} \leq r \leq s \leq R
\end{array}\right\},
$$

to find a non-increasing solution.

For simplicity of notation, in the rest of the paper we will simply denote by $\mathcal{C}$ both $\mathcal{C}_{+}(\Omega)$ and $\mathcal{C}_{-}\left(A_{R_{0}, R}\right)$, when the reasoning will be independent of the particular cone.

In both cases, thanks to the a priori estimates, we can modify $f$ at infinity in such a way to obtain a subcritical nonlinearity $\tilde{f}$. This leads us to study a new subcritical problem, with the property that all solutions of the new problem belonging to $\mathcal{C}$ solve also the original problem (1.1). The energy functional associated to the new problem is clearly well-defined in the whole $H_{\Omega, 0}^{S}$. To get a solution of the new problem belonging to $\mathcal{C}$, we prove that a mountain passtype theorem holds inside the cone $\mathcal{C}$. The main difficulty here is that we need to find a critical point of the energy, belonging to a set $(\mathcal{C})$ which is strictly smaller than the domain $\left(H_{\Omega, 0}^{s}\right)$ of the energy functional itself. To overcome this difficulty we build a deformation $\eta$ for the Deformation Lemma 4.8 which preserves the cone, cf. also Lemma 4.6. Once the minimax solution is found, we need to prove that it is non-constant. We further restrict our cone, working in a subset of $\mathcal{C}$ in which the only constant solution of (1.1) is the constant $u_{0}$ defined in $\left(f_{3}\right)$. In this set, we are able to distinguish the mountain pass solution from the constant using an energy estimate.

The multiplicity part of Theorem 1.1 can be easily obtained by repeating the same arguments around each constant solution $u_{0}$ : in case we have more than one $u_{0}$ satisfying $\left(f_{3}\right)$, for each $u_{0, i}$, we work in a subset of $\mathcal{C}$ made of functions $u$ whose image is contained in a neighborhood of $u_{0, i}$. This allows us to localize each mountain pass solution and to prove that to each $u_{0, i}$ corresponds a different solution of the problem.

The paper is organized as follows:

- In Section 2, we recall some basic properties of our nonlocal Neumann problem. In particular, we describe its variational structure and we establish a strong maximum principle;

- In Section 3, we prove the a priori bounds, both in $L^{\infty}$ and in the right energy space, which will be crucial for our existence result;

- Section 4 contains the Mountain Pass-type Theorem (Theorem 4.12) which establishes existence of a radial, non-negative, non-decreasing solution and whose main ingredient relies on a Deformation Lemma inside the cone $\mathcal{C}$ (see Lemma 4.8);

- Finally, in Section 5, we prove that the solution, found via Mountain Pass argument, is not constant. 


\section{The notion of nonlocal normal derivative and the variational structure of the problem}

In this section, we comment on the notion of nonlocal normal derivative $\mathcal{N}_{s}$ and we describe some structural properties of the nonlocal Neumann problem under consideration, with particular emphasis on its variational structure.

As mentioned in the Introduction, we use the following notion of nonlocal normal derivative:

$$
\mathcal{N}_{s} u(x):=c_{n, s} \int_{\Omega} \frac{u(x)-u(y)}{|x-y|^{n+2 s}} d y, \quad x \in \mathbb{R}^{n} \backslash \bar{\Omega} .
$$

As well explained in [11], with this notion of normal derivative, problem (1.1) has a variational structure. We emphasize that the operator $(-\Delta)^{s}$ that we consider is the standard fractional Laplacian on $\mathbb{R}^{n}$ (notice that the integration in (1.4) is taken on the whole $\mathbb{R}^{n}$ ) and not the regional one (where the integration is done only on $\Omega$ ). This choice will be reflected in the associated energy functional (see e.g. (2.12)). We note in passing that, in [1], it is shown that the fractional Laplacian $(-\Delta)^{s} u$ under homogeneous nonlocal Neumann boundary conditions $\left(\mathcal{N}_{S} u=0\right.$ in $\left.\mathbb{R}^{n} \backslash \bar{\Omega}\right)$ can be expressed as a regional operator with a kernel having logarithmic behavior at the boundary. There are other possible notions of "Neumann conditions" for problems involving fractional powers of the Laplacian (depending on which type of operator one considers), which all recover the classical Neumann condition in the limit case $s \uparrow 1$. See Setion 7 in [11] and reference therein, for a more precise discussion on possible different definitions.

The choice of the standard fractional Laplacian $(-\Delta)^{S}$ and of the corresponding normal derivative $\mathcal{N}_{S}$ has also a specific probabilistic interpretation, that is well described in Section 2 of [11]. The idea is the following. Let us a consider a particle that moves randomly in $\mathbb{R}^{n}$ according to the following law: if the particle is located at a point $x \in \mathbb{R}^{n}$, it can jump at any other point $y \in \mathbb{R}^{n}$ with a probability that is proportional to $|x-y|^{-n-2 s}$. It is well known that the probability density $u(x, t)$ that the particle is situated at the point $x$ at time $t$, solves the fractional heat equation $u_{t}+(-\Delta)^{s} u=0$. If now we replace the whole space $\mathbb{R}^{n}$ with a bounded domain $\Omega$, we need to specify what are the "boundary conditions", that is what happens when the particle exits $\Omega$. The choice of the Neumann condition $\mathcal{N}_{s} u=0$ corresponds to the following situation: when the particle reaches a point $x \in \mathbb{R}^{n} \backslash \bar{\Omega}$, it may jump back at any point $y \in \Omega$ with a probability density that, again, is proportional to $|x-y|^{-n-2 s}$. Just as a comparison, if in place of Neumann boundary conditions, one considers the more standard Dirichlet boundary conditions (that in this nonlocal setting, reads $u \equiv 0$ in $\mathbb{R}^{n} \backslash \bar{\Omega}$ ), this would correspond to killing the particle when it exits $\Omega$.

We pass now to describe some variational properties of our nonlocal Neumann problem. Let us start with an integration by part formula that justify the choice of $\mathcal{N}_{s} u$. In what follows $\Omega^{c}$ will denote the complement of $\Omega$ in $\mathbb{R}^{n}$.

Lemma 2.1 (Lemma 3.3 in [11]). Let $u$ and $v$ be bounded $C^{2}$ functions defined on $\mathbb{R}^{n}$. Then, the following formula holds 


$$
\begin{gathered}
\frac{c_{n, s}}{2} \iint_{\mathbb{R}^{2 n} \backslash\left(\Omega^{c}\right)^{2}} \frac{(u(x)-u(y))(v(x)-v(y))}{|x-y|^{n+2 s}} d x d y \\
=\int_{\Omega} v(-\Delta)^{s} u d x+\int_{\Omega^{c}} v \mathcal{N}_{s} u d x .
\end{gathered}
$$

Remark 2.2. As a consequence of Lemma 2.1, if $u \in C^{2}\left(\mathbb{R}^{n}\right)$ solves (1.1), taking $v \equiv 1$ in (2.2), we get

$$
\int_{\Omega}(-\Delta)^{s} u d x=0
$$

We now introduce the functional space where the problem is set. Let $u, v: \mathbb{R}^{n} \rightarrow \mathbb{R}$ be measurable functions, we set

$$
[u]_{H_{\Omega, 0}^{s}}:=\left(\frac{c_{n, s}}{2} \iint_{\mathbb{R}^{2 n} \backslash\left(\Omega^{c}\right)^{2}} \frac{|u(x)-u(y)|^{2}}{|x-y|^{n+2 s}} d x d y\right)^{1 / 2}
$$

and we define the space

$$
H_{\Omega, 0}^{s}:=\left\{u: \mathbb{R}^{n} \rightarrow \mathbb{R}, u \in L^{2}(\Omega):[u]_{H_{\Omega, 0}^{s}}<+\infty\right\}
$$

equipped with the scalar product

$$
(u, v)_{H_{\Omega, 0}^{s}}:=\int_{\Omega} u v d x+\frac{c_{n, s}}{2} \iint_{\mathbb{R}^{2 n} \backslash\left(\Omega^{c}\right)^{2}} \frac{(u(x)-u(y))(v(x)-v(y))}{|x-y|^{n+2 s}} d x d y,
$$

and with the induced norm

$$
\|u\|_{H_{\Omega, 0}^{s}}:=\|u\|_{L^{2}(\Omega)}+[u]_{H_{\Omega, 0}^{s}} .
$$

By [11, Proposition 3.1], we know that $\left(H_{\Omega, 0}^{s},(\cdot, \cdot)_{H_{\Omega, 0}^{s}}\right)$ is a Hilbert space.

In this paper we will mainly work with the notion of weak solutions for problem (1.1), which naturally belong to the energy space $H_{\Omega, 0}^{s}$ but, at some point (more precisely, when we will apply a strong maximum principle - see Proposition 2.6) we will need to consider also classical solutions. For this reason, let us recall under which condition the fractional Laplacian given by the expression (1.4) is well defined. Let $\mathcal{L}_{S}$ denote the following set of functions:

$$
\mathcal{L}_{s}:=\left\{u: \mathbb{R}^{n} \rightarrow \mathbb{R}: \int_{\mathbb{R}^{n}} \frac{|u(x)|}{1+|x|^{n+2 s}} d x<\infty\right\} .
$$


Let $\Omega$ be a bounded set in $\mathbb{R}^{n}, s>1 / 2$, and let $u \in \mathcal{L}_{s}$ be a $C^{1,2 s+\varepsilon-1}$ function in $\Omega$ for some $\varepsilon>0$. Then $(-\Delta)^{s} u$ is continuous on $\Omega$ and its value is given by the integral in (1.4) (see Proposition 2.4 in [16]).

In particular, the condition $u \in \mathcal{L}_{s}$ ensures integrability at infinity for the integral in (1.4). Moreover, if $u$ belongs to the energy space $H_{\Omega, 0}^{s}$, then automatically it is in $\mathcal{L}_{s}$, according to the following result.

\section{Lemma 2.3. Let $\Omega$ be a bounded set in $\mathbb{R}^{n}$. Then}

$$
H_{\Omega, 0}^{S} \subset \mathcal{L}_{s} .
$$

Proof. We prove that if $u \in H_{\Omega, 0}^{s}$, then it satisfies the integrability condition

$$
\int_{\mathbb{R}^{n}} \frac{|u(x)|^{2}}{1+|x|^{n+2 s}} d x<\infty
$$

which, in particular, implies that $u \in \mathcal{L}_{s}$, by using Hölder inequality and observing that $(1+$ $\left.|x|^{n+2 s}\right)^{-1} \in L^{1}\left(\mathbb{R}^{n}\right)$.

Throughout this proof we denote by $C$ many different positive constants whose precise value is not important for the goal of the proof and may change from time to time. Let $\Omega^{\prime}$ be a compact set contained in $\Omega$. We have

$$
\begin{aligned}
\infty & >\int_{\Omega} \int_{\mathbb{R}^{n}} \frac{|u(x)-u(y)|^{2}}{|x-y|^{n+2 s}} d x d y \\
\geq & \int_{\Omega} \int_{\Omega} \frac{|u(x)-u(y)|^{2}}{|x-y|^{n+2 s}} d x d y+\int_{\Omega^{\prime}} \int_{\mathbb{R}^{n} \backslash \Omega} \frac{|u(x)-u(y)|^{2}}{|x-y|^{n+2 s}} d x d y \\
\geq & \int_{\Omega} \int_{\Omega} \frac{|u(x)-u(y)|^{2}}{|x-y|^{n+2 s}} d x d y \\
& \quad+\frac{1}{2} \int_{\Omega^{\prime}} \int_{\mathbb{R}^{n} \backslash \Omega} \frac{|u(x)|^{2}}{|x-y|^{n+2 s}} d x d y-\int_{\Omega^{\prime}} \int_{\mathbb{R}^{n} \backslash \Omega} \frac{|u(y)|^{2}}{|x-y|^{n+2 s}} d x d y,
\end{aligned}
$$

where, in the last estimate we have used that $|a-b|^{2} \geq \frac{1}{2} a^{2}-b^{2}$ by Young inequality.

Since $u \in H_{\Omega, 0}^{s}$, clearly the first term on the r.h.s is finite. Moreover, using that for $x \in \mathbb{R}^{n} \backslash \Omega$ and $y \in \Omega^{\prime}$ one has that $|x-y| \geq \omega$, for some $\omega>0$, and the integrability of the kernel at infinity, we have for every $y \in \Omega^{\prime}$

$$
\int_{\mathbb{R}^{n} \backslash \Omega} \frac{1}{|x-y|^{n+2 s}} d x \leq C \int_{\omega}^{\infty} \tau^{n-1-(n+2 s)} d \tau=\frac{C}{\omega^{2 s}},
$$

where $C$ is independent of $y \in \Omega^{\prime}$. Hence, 


$$
\begin{aligned}
\int_{\Omega^{\prime}} \int_{\mathbb{R}^{n} \backslash \Omega} \frac{|u(y)|^{2}}{|x-y|^{n+2 s}} d x d y & \leq \int_{\Omega^{\prime}}|u(y)|^{2}\left(\int_{\mathbb{R}^{n} \backslash \Omega} \frac{1}{|x-y|^{n+2 s}} d x\right) d y \\
& \leq \frac{C}{\omega^{2 s}} \int_{\Omega^{\prime}}|u(y)|^{2} d y<\infty .
\end{aligned}
$$

Therefore, combining (2.8) with (2.9), we deduce that

$$
\int_{\Omega^{\prime}} \int_{\mathbb{R}^{n} \backslash \Omega} \frac{|u(x)|^{2}}{|x-y|^{n+2 s}} d x d y<\infty .
$$

Finally, since $\Omega$ (and thus $\Omega^{\prime}$ ) is bounded, we have that there exists some number $d$ depending only on $\Omega$ such that $|x-y| \leq d+|x|$ for every $x \in \mathbb{R}^{n} \backslash \Omega$ and $y \in \Omega^{\prime}$, which implies that

$$
\int_{\Omega^{\prime}} \int_{\mathbb{R}^{n} \backslash \Omega} \frac{|u(x)|^{2}}{|x-y|^{n+2 s}} d x d y \geq\left|\Omega^{\prime}\right| \int_{\mathbb{R}^{n} \backslash \Omega} \frac{|u(x)|^{2}}{(d+|x|)^{n+2 s}} d x .
$$

This last inequality, together with the fact that

$$
\int_{\Omega} \frac{|u(x)|^{2}}{(d+|x|)^{n+2 s}} d x<\infty
$$

(since $u \in L^{2}(\Omega)$ ) concludes the proof.

Since it will be useful later on, we introduce also some standard notation for fractional Sobolev spaces. We set

$$
[u]_{H^{s}(\Omega)}:=\left(\frac{c_{n, s}}{2} \iint_{\Omega^{2}} \frac{|u(x)-u(y)|^{2}}{|x-y|^{n+2 s}} d x d y\right)^{\frac{1}{2}} .
$$

We denote by $H^{s}(\Omega)$ the space

$$
H^{s}(\Omega):=\left\{u \in L^{2}(\Omega):[u]_{H^{s}(\Omega)}<\infty\right\},
$$

equipped with the norm

$$
\|u\|_{H^{s}(\Omega)}=\|u\|_{L^{2}(\Omega)}+[u]_{H^{s}(\Omega)} .
$$

Notice that in the definition $[u]_{H^{s}(\Omega)}$ the double integral is taken over $\Omega \times \Omega$, which differs from the seminorm defined in (2.4) related to the energy functional of our problem.

Since the following obvious inequality holds between the usual $H^{S}$-seminorm and the seminorm $[\cdot]_{H_{\Omega, 0}}$ defined in (2.4): 


$$
[u]_{H_{\Omega, 0}^{s}} \geq[u]_{H^{s}(\Omega)}
$$

as an easy consequence of the fractional compact embedding $H^{s}(\Omega) \hookrightarrow \hookrightarrow L^{q}(\Omega)$ (see for example Section 7 in [2] and remind that $H^{s}(\Omega)=W^{s, 2}(\Omega)$ ), we have the following.

Proposition 2.4. The space $H_{\Omega, 0}^{s}$ is compactly embedded in $L^{q}(\Omega)$ for every $q \in\left[1,2_{s}^{*}\right)$, where

$$
2_{s}^{*}:= \begin{cases}\frac{2 n}{n-2 s} & \text { if } 2 s<n \\ +\infty & \text { otherwise }\end{cases}
$$

is the fractional Sobolev critical exponent.

Given $h \in L^{2}(\Omega)$, we consider now the following linear problem

$$
\begin{cases}(-\Delta)^{s} u+u=h & \text { in } \Omega \\ \mathcal{N}_{s} u=0 & \text { in } \mathbb{R}^{n} \backslash \bar{\Omega}\end{cases}
$$

Definition 2.5. We say that a function $u \in H_{\Omega, 0}^{s}$ is a weak solution of problem (2.11) if

$$
\frac{c_{n, s}}{2} \iint_{\mathbb{R}^{2 n} \backslash\left(\Omega^{c}\right)^{2}} \frac{(u(x)-u(y))(v(x)-v(y))}{|x-y|^{n+2 s}} d x d y+\int_{\Omega} u v d x=\int_{\Omega} h v .
$$

With this definition one can easily see that weak solutions of problem (2.11) can be found as critical points of the following energy functional defined on the space $H_{\Omega, 0}^{s}$, cf. [11, Proposition 3.7]:

$$
\mathcal{E}(u):=\frac{c_{n, s}}{4} \iint_{\mathbb{R}^{2 n} \backslash\left(\Omega^{c}\right)^{2}} \frac{|u(x)-u(y)|^{2}}{|x-y|^{n+2 s}} d x d y+\frac{1}{2} \int_{\Omega} u^{2} d x-\int_{\Omega} h u d x .
$$

We state now a strong maximum principle for the fractional Laplacian with nonlocal Neumann conditions.

Theorem 2.6. Let $u \in C^{1,2 s+\varepsilon-1}(\Omega) \cap \mathcal{L}_{S}$ (for some $\varepsilon>0$ ) satisfy

$$
\begin{cases}(-\Delta)^{s} u \geq 0 & \text { in } \Omega, \\ u \geq 0 & \text { in } \Omega, \\ \mathcal{N}_{s} u \geq 0 & \text { in } \mathbb{R}^{n} \backslash \bar{\Omega} .\end{cases}
$$

Then, either $u>0$ or $u \equiv 0$ a.e. in $\Omega$.

Proof. Assume that $u$ is not a.e. identically zero and let us show that $u>0$ a.e. in $\Omega$. We argue by contradiction: suppose that the set in $\Omega$ on which $u$ vanishes has positive Lebesgue measure, and let call it $Z$, i.e. 


$$
Z:=\{x \in \Omega \mid u(x)=0\}, \quad \text { and } \quad|Z|>0 .
$$

Let now $\bar{x} \in Z$. Since $u$ satisfies $(-\Delta)^{s} u \geq 0$ in $\Omega$, using the definition of fractional Laplacian, we have that

$$
\begin{aligned}
\int_{\mathbb{R}^{n} \backslash \Omega} \frac{u(\bar{x})-u(y)}{|\bar{x}-y|^{n+2 s}} d y & \geq-\int_{\Omega} \frac{u(\bar{x})-u(y)}{|\bar{x}-y|^{n+2 s}} d y \\
& =\int_{\Omega} \frac{u(y)}{|\bar{x}-y|^{n+2 s}} d y>0,
\end{aligned}
$$

where the last strict inequality comes from the fact that we are assuming that $u$ is strictly positive on a subset of $\Omega$ of positive Lebesgue measure (otherwise it would be $u \equiv 0$ a.e. in $\Omega$ ).

Integrating the above inequality on the set $Z$ and using that $|Z|>0$, we deduce that

$$
\int_{Z} \int_{\mathbb{R}^{n} \backslash \Omega} \frac{u(\bar{x})-u(y)}{|\bar{x}-y|^{n+2 s}} d y d \bar{x}>0 .
$$

On the other hand, using that $u \geq 0$ in $\Omega$, we have

$$
\begin{aligned}
c_{n, s} \int_{Z} \int_{\mathbb{R}^{n} \backslash \Omega} \frac{u(\bar{x})-u(y)}{|\bar{x}-y|^{n+2 s}} d y d \bar{x} & \leq c_{n, s} \int_{\Omega} \int_{\mathbb{R}^{n} \backslash \Omega} \frac{u(x)-u(y)}{|x-y|^{n+2 s}} d y d x \\
& =-c_{n, s} \int_{\mathbb{R}^{n} \backslash \Omega} \int_{\Omega} \frac{u(y)-u(x)}{|x-y|^{n+2 s}} d x d y \\
& =-\int_{\mathbb{R}^{n} \backslash \Omega} \mathcal{N}_{s} u(y) d y \leq 0 .
\end{aligned}
$$

This contradicts (2.13) and concludes the proof.

Remark 2.7. Arguing in the same way, it is easy to see that the above strong maximum principle holds true when adding a zero order term in the equation satisfied in $\Omega$ (that is considering solutions of $(-\Delta)^{s} u(x)+c(x) u(x) \geq 0$ in $\left.\Omega\right)$.

We conclude this Section with two results of [11]. The first one gives a further justification of calling $\mathcal{N}_{S}$ a "nonlocal normal derivative".

Proposition 2.8 (Proposition 5.1 of [11]). Let $\Omega$ be any bounded Lipschitz domain of $\mathbb{R}^{n}$ and let $u$ and $v$ be $C^{2}$ functions with compact support in $\mathbb{R}^{n}$.

Then,

$$
\lim _{s \rightarrow 1} \int_{\mathbb{R}^{n} \backslash \Omega} \mathcal{N}_{s} u v d x=\int_{\partial \Omega} \partial_{\nu} u v d x,
$$

where $\partial_{\nu}$ denotes the external normal derivative to $\partial \Omega$. 
The last result that we recall from [11], describes the spectrum of the fractional Laplacian 1 with zero Neumann boundary conditions.

Theorem 2.9 (Theorem 3.11 in [11]). There exists a diverging sequence of non-negative values

$$
0=\lambda_{1}<\lambda_{2} \leq \lambda_{3} \leq \ldots,
$$

and a sequence of functions $u_{i}: \mathbb{R}^{n} \rightarrow \mathbb{R}$ such that

$$
\begin{cases}(-\Delta)^{s} u_{i}(x)=\lambda_{i} u_{i}(x) & \text { for any } x \in \Omega \\ \mathcal{N}_{s} u_{i}(x)=0 & \text { for any } x \in \mathbb{R}^{n} \backslash \bar{\Omega}\end{cases}
$$

Moreover, the functions $u_{i}$ (restricted to $\Omega$ ) provide a complete orthogonal system in $L^{2}(\Omega)$.

\section{A priori bounds for monotone radial solutions}

Without loss of generality, from now on we suppose that $f$ satisfies the further assumption

$\left(f_{0}\right) \quad f(t) \geq 0$ and $f^{\prime}(t) \geq 0$ for every $t \in[0, \infty)$.

If this is not the case, it is always possible to reduce problem (1.1) to an equivalent one having a non-negative and non-decreasing nonlinearity, cf. [8, Lemma 2.1].

We look for solutions to (1.1) in the cone $\mathcal{C}$ defined in (1.6) and (1.7).

It is easy to prove that $\mathcal{C}$ is a closed convex cone in $H_{\Omega, 0}^{s}$, i.e., the following properties hold for all $u, v \in \mathcal{C}$ and $\lambda \geq 0$ :

(i) $\lambda u \in \mathcal{C}$;

(ii) $u+v \in \mathcal{C}$;

(iii) if also $-u \in \mathcal{C}$, then $u \equiv 0$;

(iv) $\mathcal{C}$ is closed in the $H^{s}$-topology.

We will use the above properties of $\mathcal{C}$ in Lemma 4.8 .

We state now an embedding result for radial functions belonging to fractional Sobolev spaces, which can be found in [15] (see also [6]).

Lemma 3.1. If $s>1 / 2$ and $0<\bar{R}<R$, there exists a positive constant $C_{\bar{R}}=C_{\bar{R}}(\bar{R}, n, s)$ such that

$$
\|u\|_{L^{\infty}\left(B_{R} \backslash B_{\bar{R}}\right)} \leq C_{\bar{R}}\|u\|_{H_{B_{R} \backslash B_{\bar{R}}, 0}^{s}}
$$

for all $u$ radial in $H_{B_{R} \backslash B_{\bar{R}}, 0}^{S}$

Proof. The proof is the same as in [6, Lemma 4.3], we report it here for the sake of completeness. Let $\bar{R}<\rho<R$. Using that $u$ is radial, $s>1 / 2$, and the trace inequality for $H^{s}\left(B_{\rho} \backslash B_{\bar{R}}\right.$ ) (see e.g. [17, Section 3.3.3]), we have for every $x \in \partial B_{\rho}$ 


$$
\begin{aligned}
|u(x)|^{2} & =\frac{\rho^{1-n}}{n \omega_{n}} \int_{\partial B_{\rho}} u^{2} d \mathcal{H}^{n-1} \\
& \leq C \frac{\rho^{1-n}}{n \omega_{n}} \rho^{2 s-1}\left\{[u]_{H^{s}\left(B_{\rho} \backslash B_{\bar{R}}\right)}^{2}+\frac{1}{\rho^{2 s}}\|u\|_{L^{2}\left(B_{\rho} \backslash B_{\bar{R}}\right)}^{2}\right\},
\end{aligned}
$$

where $\omega_{n}$ is the volume of the unit sphere in $\mathbb{R}^{n}$ and $d \mathcal{H}^{n-1}$ denotes the $(n-1)$-dimensional Hausdorff measure. We immediately deduce that for every $x \in \partial B_{\rho}$

$$
\begin{aligned}
|u(x)| & \leq \begin{cases}C|x|^{-\frac{n-2 s}{2}}\|u\|_{H^{s}\left(B_{\rho} \backslash B_{\bar{R}}\right)} & \text { if } \rho=|x| \geq 1, \\
C \frac{|x|^{-\frac{n-2 s}{2}}}{\rho^{s}}\|u\|_{H^{s}\left(B_{\rho} \backslash B_{\bar{R}}\right)} & \text { if } \rho=|x|<1\end{cases} \\
& \leq C|x|^{-\frac{n-2 s}{2}}\left(1+\frac{1}{\rho^{s}}\right)\|u\|_{H^{s}\left(B_{\rho} \backslash B_{\bar{R}}\right)} \\
& \leq C \bar{R}^{-\frac{n-2 s}{2}}\left(1+\bar{R}^{-s}\right)\|u\|_{H^{s}\left(B_{R} \backslash B_{\bar{R}}\right)}
\end{aligned}
$$

Hence, we conclude that

$$
\|u\|_{L^{\infty}\left(B_{R} \backslash B_{\bar{R}}\right)} \leq C \bar{R}^{-\frac{n-2 s}{2}}\left(1+\bar{R}^{-s}\right)\|u\|_{H^{s}\left(B_{R} \backslash B_{\bar{R}}\right)}
$$$$
\leq C \bar{R}^{-\frac{n-2 s}{2}}\left(1+\bar{R}^{-s}\right)\|u\|_{H_{B_{R} \backslash B_{\bar{R}}, 0}^{s}},
$$

which proves the statement, with $C_{\bar{R}}:=C \bar{R}^{-\frac{n-2 s}{2}}\left(1+\bar{R}^{-s}\right)$.

As mentioned above, working in the cones $\mathcal{C}$ of non-negative, radial and monotone functions has the advantage to have an a priori $L^{\infty}$ bound, according to the following lemma. In particular, from the proof of the next lemma it will be clear the role of the non-decreasing monotonicity in the case of the ball.

Lemma 3.2. Let $s>1 / 2$ and $\Omega$ be the ball $B_{R}$ or the annulus $A_{R_{0}, R}$ as in (1.2), (1.3). There exists a constant $C=C\left(R, R_{0}, n, s\right)>0$ such that

$$
\|u\|_{L^{\infty}(\Omega)} \leq C\|u\|_{H_{\Omega, 0}^{s}} \quad \text { for all } u \in \mathcal{C} .
$$

Proof. Case $\Omega=B_{R}$. In this case, $\mathcal{C}=\mathcal{C}_{+}\left(B_{R}\right)$. Since $u$ is radial and non-decreasing, we have that $\|u\|_{L^{\infty}(\Omega)}=\|u\|_{L^{\infty}\left(B_{R} \backslash B_{R / 2}\right)}$. Hence, the conclusion follows by (3.1), observing that here $\bar{R}=R / 2>0$.

Case $\Omega=A_{R_{0}, R}$. In the annulus, the same proof as before works both for $u \in \mathcal{C}_{+}$and for $u \in \mathcal{C}_{-}$. We observe that in this case the constant $C$ depends on $R_{0}$ (and not on $R$ ).

Thanks to the previous lemma, it would be enough to restrict the energy functional to $\mathcal{C}$ to get $\mathcal{C}$-constrained critical points; this is the approach in [14]. Nonetheless, as well explained in [14], the cone $\mathcal{C}$ has empty interior in the $H^{s}$-topology, as a consequence it does not contain enough test functions to guarantee that constrained critical points are indeed free critical points. 
Hence, form $\mathfrak{F}_{M, \delta}$.

$$
f(t) \geq(1+\delta) t \quad \text { for all } t \geq M .
$$

The existence of $\delta, M>0$ follows by $\left(f_{2}\right)$. We introduce the following set of functions

$$
\mathfrak{F}_{M, \delta}:=\{g \in C([0, \infty)): g \geq 0, \quad g(t) \geq(1+\delta) t \text { for all } t \geq M\} .
$$

We remark that $\mathfrak{F}_{M, \delta}$ depends on $f$ only through $\delta$ and $M$. In the remaining part of this section, we shall derive some a priori estimates which are uniform in $\mathfrak{F}_{M, \delta}$ and hence depend only on $M$ and $\delta$, and not on the specific function $g$ belonging to $\mathfrak{F}_{M, \delta}$. This will be useful in the rest of the paper, since we will deal with a truncated function.

We give now the definition of weak solution for a general nonlinear Neumann problem of the

$$
\begin{cases}(-\Delta)^{s} u+u=g(u) & \text { in } \Omega \\ u \geq 0 & \text { in } \Omega \\ \mathcal{N}_{s} u=0 & \text { in } \mathbb{R}^{n} \backslash \bar{\Omega} .\end{cases}
$$

Definition 3.3. We say that a non-negative function $u \in H_{\Omega, 0}^{s}$ is a weak solution of problem (3.4) if for every $v \in H_{\Omega, 0}^{s}$

$$
\frac{c_{n, s}}{2} \iint_{\mathbb{R}^{2 n} \backslash\left(\Omega^{c}\right)^{2}} \frac{(u(x)-u(y))(v(x)-v(y))}{|x-y|^{n+2 s}} d x d y+\int_{\Omega} u v d x=\int_{\Omega} g(u) v .
$$

The following Lemma gives an $L^{1}$ bound for solutions to (3.4) with $g$ belonging to the class

Lemma 3.4. Let $g$ be any function in $\mathfrak{F}_{M, \delta}$. Then, there exists a constant $K_{1}=K_{1}(R, n, M, \delta)>0$ such that any weak solution $u \in \mathcal{C}$ of (3.4) satisfies

$$
\|u\|_{L^{1}(\Omega)} \leq K_{1} .
$$

Proof. Testing the notion of weak solution with $v \equiv 1$ and using that $g \in \mathfrak{F}_{M, \delta}$, we get

$$
\int_{\Omega} u d x=\int_{\{u<M\}} g(u) d x+\int_{\{u \geq M\}} g(u) d x \geq(1+\delta) \int_{\{u \geq M\}} u d x .
$$




$$
M|\Omega| \geq \int_{\{u<M\}} u d x \geq \delta \int_{\{u \geq M\}} u d x
$$

and so

$$
\int_{\Omega} u d x=\int_{\{u<M\}} u d x+\int_{\{u \geq M\}} u d x \leq M|\Omega|\left(1+\frac{1}{\delta}\right)=: K_{1} .
$$

The following lemma gives a uniform a priori bound in $L^{\infty}$ for solutions belonging to the cone $\mathcal{C}$ of problems (3.4), with $g \in \mathfrak{F}_{M, \delta}$.

Lemma 3.5. There exist two positive constants $K_{\infty}=K_{\infty}\left(R_{0}, R, n, s, M, \delta\right)$ and $K_{2}={ }_{13}$ $K_{2}\left(R_{0}, R, n, s, M, \delta\right)$, such that for any $u \in \mathcal{C}$ weak solution of problem (3.4), the following 14 estimates hold:

$$
\|u\|_{L^{\infty}(\Omega)} \leq K_{\infty} \quad \text { and } \quad\|u\|_{H_{\Omega, 0}^{s}} \leq K_{2}
$$

Proof. Choosing again $v \equiv 1$ in the definition of weak solution, we have

$$
\int_{\Omega} u d x=\int_{\Omega} g(u) d x .
$$

On the other hand, testing the equation with $u$ itself and using Lemma 3.2, we deduce

$$
\begin{aligned}
\|u\|_{L^{\infty}(\Omega)}^{2} & \leq C^{2}\left(\iint_{\mathbb{R}^{2 n} \backslash\left(\Omega^{c}\right)^{2}} \frac{|u(x)-u(y)|^{2}}{|x-y|^{n+2 s}} d x d y+\int_{\Omega} u^{2} d x\right) \\
& =C^{2} \int_{\Omega} g(u) u d x \leq C^{2}\|u\|_{L^{\infty}(\Omega)} \int_{\Omega} g(u) d x .
\end{aligned}
$$

Combining (3.5) with the previous estimate, we conclude that

$$
\|u\|_{L^{\infty}(\Omega)} \leq C^{2}\|u\|_{L^{1}(\Omega)} \leq C^{2} K_{1}=: K_{\infty}
$$

where the last estimate comes from Lemma 3.4. Finally, this bound on $\|u\|_{L^{\infty}(\Omega)}$ combined with 38 inequality (3.6) above, gives the following uniform bound on $\|u\|_{H_{\Omega, 0}^{s}}$ : 39

$$
\|u\|_{H_{\Omega, 0}^{s}}^{2} \leq\|u\|_{L^{\infty}(\Omega)} \int g(u) d x=\|u\|_{L^{\infty}(\Omega)}\|u\|_{L^{1}(\Omega)} \leq C^{2} K_{1}^{2}=: K_{2}^{2} . \quad \square \quad 41
$$

We now prove a regularity result for weak solutions of (1.1) belonging to the cone $\mathcal{C}$. $\quad 45$

Lemma 3.6. Let $u \in \mathcal{C}$ be a weak solution of (1.1). Then $u \in C^{2}\left(\mathbb{R}^{n}\right)$. 
Proof. By Lemma 3.5 we know that $u \in L^{\infty}(\Omega)$. Furthermore, by the nonlocal Neumann boundary conditions, we have that

$$
u(x)=\frac{\int_{\Omega} \frac{u(y)}{|x-y|^{n+2 s}} d y}{\int_{\Omega} \frac{1}{|x-y|^{n+2 s}} d y} \text { for every } x \in \mathbb{R}^{n} \backslash \bar{\Omega} .
$$

Thus, for any $\varepsilon>0$ we get for every $x \in \mathbb{R}^{n} \backslash \Omega_{\varepsilon}:=\left\{x \in \mathbb{R}^{n}: \operatorname{dist}(x, \Omega) \geq \varepsilon\right\}$

$$
u(x)=\frac{\int_{\Omega} \frac{u(y)}{|x-y|^{n+2 s}} d y}{\int_{\Omega} \frac{1}{|x-y|^{n+2 s}} d y} \leq\|u\|_{L^{\infty}(\Omega)} \frac{\int_{\Omega} \frac{1}{|x-y|^{n+2 s}} d y}{\int_{\Omega} \frac{1}{|x-y|^{n+2 s}} d y}=\|u\|_{L^{\infty}(\Omega)} .
$$

Therefore, being this estimate uniform in $\varepsilon$, we get $|u(x)| \leq\|u\|_{L^{\infty}(\Omega)}$ for every $x \in \mathbb{R}^{n} \backslash \bar{\Omega}$. Hence, $u \in L^{\infty}\left(\mathbb{R}^{n}\right)$ and so, using [16, Proposition 2.9] with $w=f(u)-u \in L^{\infty}\left(\mathbb{R}^{n}\right)$, we obtain $u \in C^{1, \alpha}\left(\mathbb{R}^{n}\right)$ for every $\alpha \in(0,2 s-1)$. Then, recalling that $f \in C^{1, \gamma}$, we can use a bootstrap argument, and apply [16, Proposition 2.8] to conclude the proof.

\section{Existence of a mountain pass radial solution}

In this section we prove the existence of a radial solution of (1.1) via a Mountain Pass-type Theorem. We are now ready to start the truncation method described in the Introduction: we will modify $f$ in $\left(K_{\infty},+\infty\right)$, where $K_{\infty}$ is the $L^{\infty}$ bound given in Lemma 3.5, in such a way to have a subcritical nonlinearity $\tilde{f}$.

Lemma 4.1. For every $\ell \in\left(2,2_{s}^{*}\right)$, there exists $\tilde{f} \in \mathfrak{F}_{M, \delta} \cap C^{1}([0, \infty))$, satisfying $\left(f_{0}\right)-\left(f_{3}\right)$,

$$
\lim _{t \rightarrow \infty} \frac{\tilde{f}(t)}{t^{\ell-1}}=1,
$$

and with the property that if $u \in \mathcal{C}$ solves

$$
\begin{cases}(-\Delta)^{s} u+u=\tilde{f}(u) & \text { in } \Omega, \\ u>0 & \text { in } \Omega, \\ \mathcal{N}_{s} u=0 & \text { in } \mathbb{R}^{n} \backslash \bar{\Omega},\end{cases}
$$

then $u$ solves (1.1).

For the proof of the above lemma, we refer the reader to [4, Lemma 4.3].

As a consequence of the previous lemma, condition $\left(f_{1}\right)$, and the regularity of $f$, there exists $C>0$ for which 


$$
\tilde{f}(t) \leq C\left(1+t^{\ell-1}\right) \quad \text { for all } t \geq 0,
$$

where $\ell \in\left(2,2_{s}^{*}\right)$.

From now on in the paper, we consider the trivial extension of $\tilde{f}$, still denoted with the same symbol

$$
\tilde{f}= \begin{cases}\tilde{f} & \text { in }[0,+\infty) \\ 0 & \text { in }(-\infty, 0)\end{cases}
$$

Recalling the Definition 3.3 of weak solution (applied here with $g=\tilde{f}$ ) one can easily see that weak solutions of problem (4.2) can be found as critical points of the following energy functional defined on the space $H_{\Omega, 0}^{s}$ :

$$
\mathcal{E}(u):=\frac{c_{n, s}}{4} \iint_{\mathbb{R}^{2 n} \backslash\left(\Omega^{c}\right)^{2}} \frac{|u(x)-u(y)|^{2}}{|x-y|^{n+2 s}} d x d y+\frac{1}{2} \int_{\Omega} u^{2} d x-\int_{\Omega} \tilde{F}(u) d x,
$$

where $\tilde{F}(t):=\int_{0}^{t} \tilde{f}(\tau) d \tau$. The proof of this fact follows from the argument in the proof of ${ }_{19}^{18}$ Proposition 3.7 in [11], with the obvious modifications due the presence of the nonlinearity $\tilde{f}$. 20

Because of (4.1) and the Sobolev embedding, the functional $\mathcal{E}$ is well defined and of class $C^{2}, \quad 21$ being $s>1 / 2$.

Lemma 4.2 (Palais-Smale condition). The functional $\mathcal{E}$ satisfies the Palais-Smale condition, i.e. 24 every $(P S)$-sequence $\left(u_{k}\right) \subset H_{\Omega, 0}^{s}$, namely a sequence satisfying

$$
\left(\mathcal{E}\left(u_{k}\right)\right) \text { is bounded and } \mathcal{E}^{\prime}\left(u_{k}\right) \rightarrow 0 \text { in }\left(H_{\Omega, 0}^{s}\right)^{*},
$$

admits a convergent subsequence.

Proof. Reasoning as in [8, Lemma 3.3], as a consequence of (4.1), there exist $\mu \in(2, \ell]$ and $T_{0}>0$ such that

$$
\tilde{f}(t) t \geq \mu \tilde{F}(t) \quad \text { for all } t \geq T_{0} .
$$

Now, let $\left(u_{k}\right) \subset H_{\Omega, 0}^{s}$ be a (PS)-sequence for $\mathcal{E}$ as in the statement. We estimate

$$
\begin{aligned}
\mathcal{E}\left(u_{k}\right)-\frac{1}{\mu} \mathcal{E}^{\prime}\left(u_{k}\right)\left[u_{k}\right] \geq & \frac{c_{n, s}}{2}\left(\frac{1}{2}-\frac{1}{\mu}\right)\left\|u_{k}\right\|_{H_{\Omega, 0}^{s}}^{2} \\
& +\int_{\left\{u_{k} \leq T_{0}\right\}}\left(\frac{1}{\mu} \tilde{f}\left(u_{k}\right) u_{k}-\tilde{F}\left(u_{k}\right)\right) d x
\end{aligned}
$$

and, being $\left(u_{k}\right)$ a $(\mathrm{PS})$-sequence,

$$
\mathcal{E}\left(u_{k}\right)-\frac{1}{\mu} \mathcal{E}^{\prime}\left(u_{k}\right)\left[u_{k}\right] \leq\left|\mathcal{E}\left(u_{k}\right)\right|+\frac{1}{\mu}\left\|\mathcal{E}^{\prime}\left(u_{k}\right)\right\|_{*}\left\|u_{k}\right\|_{H_{\Omega, 0}^{s}} \leq C\left(1+\left\|u_{k}\right\|_{H_{\Omega, 0}^{s}}\right)
$$


for some $C>0$, where we have denoted by $\|\cdot\|_{*}$ the norm of the dual space of $H_{\Omega, 0}^{s}$. Since we know that $\int_{\left\{u_{k} \leq T_{0}\right\}}\left(\frac{1}{\mu} \tilde{f}\left(u_{k}\right) u_{k}-\tilde{F}\left(u_{k}\right)\right) d x$ is uniformly bounded in $k$, we get

$$
\left(\frac{1}{2}-\frac{1}{\mu}\right)\left\|u_{k}\right\|_{H_{\Omega, 0}^{s}}^{2} \leq C\left(1+\left\|u_{k}\right\|_{H_{\Omega, 0}^{s}}\right) .
$$

Therefore, $\left(u_{k}\right)$ is bounded in $H_{\Omega, 0}^{s}$ and so there exists $u \in H_{\Omega, 0}^{s}$ such that $u_{k} \rightarrow u$ in $H_{\Omega, 0}^{s}$, up to a subsequence. By compact embedding (Proposition 2.4), $u_{k} \rightarrow u$ in $L^{\ell}(\Omega)$ and, up to a subsequence, $u_{k} \rightarrow u$ a.e. in $\Omega$. Again, since $\left(u_{k}\right)$ is a (PS)-sequence

$$
\left|\mathcal{E}^{\prime}\left(u_{k}\right)\left[u_{k}-u\right]\right| \leq\left\|\mathcal{E}^{\prime}\left(u_{k}\right)\right\|_{*}\left\|u_{k}-u\right\|_{H_{\Omega, 0}^{s}} \rightarrow 0 \quad \text { as } k \rightarrow \infty .
$$

On the other hand, by Hölder's inequality and (4.3),

$$
\begin{aligned}
\int_{\Omega} \tilde{f}\left(u_{k}\right)\left(u_{k}-u\right) d x & \leq C \int_{\Omega}\left(1+u_{k}^{\ell-1}\right)\left(u_{k}-u\right) d x \\
& \leq C\left\|1+u_{k}\right\|_{L^{\ell}(\Omega)}^{\ell-1}\left\|u_{k}-u\right\|_{L^{\ell}(\Omega)} \rightarrow 0 \quad \text { as } k \rightarrow \infty
\end{aligned}
$$

and

$$
\int_{\Omega} u_{k}\left(u_{k}-u\right) d x=\int_{\Omega}\left(u_{k}-u\right)^{2} d x+\int_{\Omega} u\left(u_{k}-u\right) d x \rightarrow 0 \quad \text { as } k \rightarrow \infty .
$$

Recalling that

$$
\begin{aligned}
\mathcal{E}^{\prime}\left(u_{k}\right)\left[u_{k}-u\right] & \\
= & \frac{c_{n, s}}{2} \iint_{\mathbb{R}^{2 n} \backslash\left(\Omega^{c}\right)^{2}} \frac{\left(u_{k}(x)-u_{k}(y)\right)\left[\left(u_{k}-u\right)(x)-\left(u_{k}-u\right)(y)\right]}{|x-y|^{n+2 s}} d x d y \\
& +\int_{\Omega} u_{k}\left(u_{k}-u\right) d x-\int_{\Omega} \tilde{f}\left(u_{k}\right)\left(u_{k}-u\right) d x,
\end{aligned}
$$

by (4.6), we have in view of (4.7) and (4.8)

$$
\lim _{\substack{k \rightarrow \infty \\ \mathbb{R}^{2 n} \backslash\left(\Omega^{c}\right)^{2}}} \iint_{\substack{u^{2}\\}} \frac{\left(u_{k}(x)-u_{k}(y)\right)\left[\left(u_{k}-u\right)(x)-\left(u_{k}-u\right)(y)\right]}{|x-y|^{n+2 s}} d x d y=0 .
$$

We claim that (4.9) implies the following

$$
\lim _{k \rightarrow \infty} \iint_{\mathbb{R}^{2 n} \backslash\left(\Omega^{c}\right)^{2}} \frac{\left|u_{k}(x)-u_{k}(y)\right|^{2}}{|x-y|^{n+2 s}} d x d y=\iint_{\mathbb{R}^{2 n} \backslash\left(\Omega^{c}\right)^{2}} \frac{|u(x)-u(y)|^{2}}{|x-y|^{n+2 s}} d x d y .
$$


Indeed, by weak lower semicontinuity

$$
\iint_{\mathbb{R}^{2 n} \backslash\left(\Omega^{c}\right)^{2}} \frac{|u(x)-u(y)|^{2}}{|x-y|^{n+2 s}} d x d y \leq \liminf _{k \rightarrow \infty} \iint_{\mathbb{R}^{2 n} \backslash\left(\Omega^{c}\right)^{2}} \frac{\left|u_{k}(x)-u_{k}(y)\right|^{2}}{|x-y|^{n+2 s}} d x d y .
$$

Moreover, setting

$$
a:=u(x)-u(y) \quad \text { and } \quad b:=u_{k}(x)-u_{k}(y) \text {, }
$$

using the easy inequality $a^{2}+2 b(b-a) \geq b^{2}$, we deduce

$$
\begin{aligned}
\iint_{\mathbb{R}^{2 n} \backslash\left(\Omega^{c}\right)^{2}} & \frac{|u(x)-u(y)|^{2}}{|x-y|^{n+2 s}} d x d y \\
& +2 \iint_{\mathbb{R}^{2 n} \backslash\left(\Omega^{c}\right)^{2}} \frac{\left(u_{k}(x)-u_{k}(y)\right)\left(u_{k}(x)-u_{k}(y)-u(x)+u(y)\right)}{|x-y|^{n+2 s}} d x d y \\
& \geq \iint_{\mathbb{R}^{2 n} \backslash\left(\Omega^{c}\right)^{2}} \frac{\left|u_{k}(x)-u_{k}(y)\right|^{2}}{|x-y|^{n+2 s}} d x d y .
\end{aligned}
$$

Thus, by (4.9), we obtain

$$
\iint_{\mathbb{R}^{2 n} \backslash\left(\Omega^{c}\right)^{2}} \frac{|u(x)-u(y)|^{2}}{|x-y|^{n+2 s}} d x d y \geq \limsup _{k \rightarrow \infty} \iint_{\mathbb{R}^{2 n} \backslash\left(\Omega^{c}\right)^{2}} \frac{\left|u_{k}(x)-u_{k}(y)\right|^{2}}{|x-y|^{n+2 s}} d x d y,
$$

which, together with (4.11), proves the claim. Combining (4.10) with the convergence of $L^{2}$ norms $\left\|u_{k}\right\|_{L^{2}(\Omega)}^{2} \rightarrow\|u\|_{L^{2}(\Omega)}^{2}$, we get

$$
\left\|u_{k}\right\|_{H_{\Omega, 0}^{s}} \rightarrow\|u\|_{H_{\Omega, 0}^{s}} .
$$

Finally, since we also have weak convergence $u_{k} \rightarrow u$ in $H_{\Omega, 0}^{s}$, we conclude that $u_{k} \rightarrow u$ in $H_{\Omega, 0}^{S}$.

Remark 4.3. We observe that, as already noticed in [4, Remark 4.13], the truncation method (cf. Lemma 4.1) and the preliminary a priori estimate (cf. Lemma 3.5) are needed to get the subcritical growth of the nonlinearity (4.3) and the Ambrosetti-Rabinowitz condition (4.5). If the original nonlinearity $f$ of problem (1.1) satisfies those further assumptions, it is possible to skip the first part concerning a priori estimates and truncation, and to prove directly the existence of both a non-decreasing and a non-increasing (also for the ball) solutions, just starting from Lemma 4.2 with $\tilde{f}=f$. 
We define

$$
\begin{aligned}
& u_{-}:=\sup \left\{t \in\left[0, u_{0}\right): \tilde{f}(t)=t\right\} \\
& u_{+}:=\inf \left\{t \in\left(u_{0},+\infty\right): \tilde{f}(t)=t\right\}
\end{aligned}
$$

Since $\tilde{f}$ is a truncation of $f$, using Lemma 4.1 and the properties satisfied by $f$, we have that $\tilde{f}\left(u_{0}\right)=u_{0}$ and $\tilde{f}^{\prime}\left(u_{0}\right)>0$, so that $u_{0}$ is an isolated zero of the function $\tilde{f}(t)-t$. Hence,

$$
u_{-} \neq u_{0} \quad \text { and } \quad u_{+} \neq u_{0} .
$$

We point out that $u_{+}=+\infty$ is possible. Next, in order to localize the solutions, as already explained in the Introduction, we define the restricted cones

$$
\begin{aligned}
& \mathcal{C}_{+, *}:=\left\{u \in \mathcal{C}_{+}: u_{-} \leq u \leq u_{+} \text {in } \Omega\right\} \\
& \mathcal{C}_{-, *}:=\left\{u \in \mathcal{C}_{-}: u_{-} \leq u \leq u_{+} \text {in } A_{R_{0}, R}\right\}
\end{aligned}
$$

As for $\mathcal{C}$, when it will not be relevant to distinguish between the two cones $\mathcal{C}_{+, *}$ and $\mathcal{C}_{-, *}$, we $\quad 18$ will simply denote by $\mathcal{C}_{*}$ either of them

$$
\mathcal{C}_{*}:=\left\{u \in \mathcal{C}: u_{-} \leq u \leq u_{+} \text {in } \Omega\right\}
$$

Clearly, $\mathcal{C}_{*}$ is closed and convex.

Corollary 4.4. Let $c \in \mathbb{R}$ be such that $\mathcal{E}^{\prime}(u) \neq 0$ for all $u \in \mathcal{C}_{*}$ with $\mathcal{E}(u)=c$. Then, there exist two positive constants $\bar{\varepsilon}$ and $\bar{\delta}$ such that the following inequality holds

$$
\left\|\mathcal{E}^{\prime}(u)\right\|_{*} \geq \bar{\delta} \quad \text { for all } u \in \mathcal{C}_{*} \text { with }|\mathcal{E}(u)-c| \leq 2 \bar{\varepsilon} .
$$

Proof. The proof follows by Lemma 4.2. Indeed, suppose by contradiction that the thesis does not hold, then we can find a sequence $\left(u_{k}\right) \subset \mathcal{C}_{*}$ such that $\left\|\mathcal{E}^{\prime}\left(u_{k}\right)\right\|_{*}<\frac{1}{k}$ and $c-\frac{1}{k} \leq \mathcal{E}\left(u_{k}\right) \leq$ $c+\frac{1}{k}$ for all $k$. Hence, $\left(u_{k}\right)$ is a Palais-Smale sequence, and since $\mathcal{E}$ satisfies the Palais-Smale condition, up to a subsequence, $u_{k} \rightarrow u$ in $H_{\Omega, 0}^{s}$. Since $\left(u_{k}\right) \subset \mathcal{C}_{*}$ and $\mathcal{C}_{*}$ is closed, $u \in \mathcal{C}_{*}$. The fact that $\mathcal{E}$ is of class $C^{1}$ then gives $\mathcal{E}\left(u_{k}\right) \rightarrow c=\mathcal{E}(u)$ and $\mathcal{E}^{\prime}\left(u_{k}\right) \rightarrow 0=\mathcal{E}^{\prime}(u)$, which contradicts the hypothesis.

We define the operator $T:\left(H_{\Omega, 0}^{s}\right)^{*} \rightarrow H_{\Omega, 0}^{s}$ as

$$
T(h)=v, \quad \text { where } v \text { solves } \quad\left(P_{h}\right) \begin{cases}(-\Delta)^{s} v+v=h & \text { in } \Omega, \\ \mathcal{N}_{s} v=0 & \text { in } \mathbb{R} \backslash \bar{\Omega} .\end{cases}
$$

The associated energy of $\left(P_{h}\right)$, given by (2.12), is strictly convex, coercive and weakly lower semicontinuous, hence problem $\left(P_{h}\right)$ admits a unique weak solution $v \in H_{\Omega, 0}^{s}$, which is a minimizer of the energy. Hence, the definition of $T$ is well posed and

$$
T \in C\left(\left(H_{\Omega, 0}^{s}\right)^{*} ; H_{\Omega, 0}^{s}\right),
$$


(see for instance the proof Theorem 3.9 in [11]).

We introduce also the operator

$$
\tilde{T}: H_{\Omega, 0}^{s} \rightarrow H_{\Omega, 0}^{s} \quad \text { defined by } \quad \tilde{T}(u)=T(\tilde{f}(u)),
$$

with $T$ given in (4.15). Being $\ell<2_{s}^{*}, u \in H_{\Omega, 0}^{s}$ implies $u \in L^{\ell}(\Omega)$. Hence, by (4.3), $\tilde{f}(u) \in$ $L^{\ell^{\prime}}(\Omega) \subset\left(H_{\Omega, 0}^{s}\right)^{*}$ and $\tilde{T}$ is well defined.

Proposition 4.5. The operator $\tilde{T}$ is compact, i.e. it maps bounded subsets of $H_{\Omega, 0}^{s}$ into precompact subsets of $H_{\Omega, 0}^{s}$.

The proof of the previous proposition is the same as for [8, Proposition 3.2] with the obvious changes due to the different space we are working in, so we omit it.

In the following lemma we prove that the operator $\tilde{T}$ preserves the cone $\mathcal{C}_{*}$, which in turn will be useful, in Lemma 4.8, to build a deformation that preserves the cone. As mentioned in the Introduction, this is crucial to guarantee existence of a minimax solution in $\mathcal{C}_{*}$.

\section{Lemma 4.6. The operator $\tilde{T}$ defined in (4.17) satisfies $\tilde{T}\left(\mathcal{C}_{*}\right) \subseteq \mathcal{C}_{*}$.}

Proof. We first note that $u \in \mathcal{C}_{*}$ implies $\tilde{f}(u) \in \mathcal{C}$, by the properties of $\tilde{f}$. Now, let $u \in \mathcal{C}_{*}$ and $v:=\tilde{T}(u)$. We see that $v \geq 0$ in $\Omega$. Indeed, denoting by $v^{+}$the positive part of $v$, by an easy observation we have that $\left|v^{+}(x)-v^{+}(y)\right| \leq|v(x)-v(y)|$, and hence $\mathcal{E}\left(v^{+}\right) \leq \mathcal{E}(v)$. Furthermore, due to uniqueness, $v$ is radial. For the monotonicity, we distinguish the two cases.

Case $u \in \mathcal{C}_{+, *}$. In this case, we have to prove that $v$ is non-decreasing. It is enough to show that for every $r \in\left(R_{0}, R\right)$ one of the following cases occurs:

(a) $v(t) \leq v(r)$ for all $t \in\left(R_{0}, r\right)$,

(b) $v(t) \geq v(r)$ for all $t \in(r, R)$.

Indeed, if $v(\bar{t})>v(r)$ for some $R_{0}<\bar{t}<r$, by the continuity of $v$, there exists $t \in(\bar{t}, r)$ for which $v(\bar{t})>v(t)>v(r)$ which violates both $(a)$ and $(b)$. Now, we fix $r \in\left(R_{0}, R\right)$. If $\tilde{f}(u(r)) \leq v(r)$, we consider the test function

$$
\varphi_{+}(x):= \begin{cases}(v(|x|)-v(r))^{+} & \text {if } R_{0}<|x| \leq r \\ 0 & \text { otherwise }\end{cases}
$$


We have

$$
\begin{aligned}
& \iiint_{\mathbb{R}^{2 n} \backslash\left(\left(B_{r} \backslash B_{R_{0}}\right)^{c}\right)^{2}} \frac{(v(x)-v(y))\left(\varphi_{+}(x)-\varphi_{+}(y)\right)}{|x-y|^{n+2 s}} d x d y \\
& +\int_{B_{r} \backslash B_{R_{0}}} v(x) \varphi_{+}(x) d x \\
= & \int_{B_{r} \backslash B_{R_{0}}} \tilde{f}(u(x)) \varphi_{+}(x) d x \leq \tilde{f}(u(r)) \int_{B_{r} \backslash B_{R_{0}}} \varphi_{+}(x) d x \\
\leq & v(r) \int_{B_{r} \backslash B_{R_{0}}} \varphi_{+}(x) d x .
\end{aligned}
$$

Using again the definition of $\varphi_{+}$, we obtain

$$
\begin{aligned}
\iint_{\mathbb{R}^{2 n} \backslash\left(\left(B_{r} \backslash B_{R_{0}}\right)^{c}\right)^{2}} & \frac{(v(x)-v(y))\left(\varphi_{+}(x)-\varphi_{+}(y)\right)}{|x-y|^{n+2 s}} d x d y \\
& \geq \iiint_{\mathbb{R}^{2 n} \backslash\left(\left(B_{r} \backslash B_{R_{0}}\right)^{c}\right)^{2}} \frac{\left|\varphi_{+}(x)-\varphi_{+}(y)\right|^{2}}{|x-y|^{n+2 s}} d x d y .
\end{aligned}
$$

Hence, by (4.18) and (4.19)

$$
0 \geq
$$

$$
\begin{aligned}
& \iint_{\mathbb{R}^{2 n} \backslash\left(\left(B_{r} \backslash B_{R_{0}}\right)^{c}\right)^{2}} \frac{\left|\varphi_{+}(x)-\varphi_{+}(y)\right|^{2}}{|x-y|^{n+2 s}} d x d y+\int_{B_{r} \backslash B_{R_{0}}}(v(x)-v(r)) \varphi_{+}(x) d x \\
= & \iint_{\mathbb{R}^{2 n} \backslash\left(\left(B_{r} \backslash B_{R_{0}}\right)^{c}\right)^{2}} \frac{\left|\varphi_{+}(x)-\varphi_{+}(y)\right|^{2}}{|x-y|^{n+2 s}} d x d y+\int_{B_{r} \backslash B_{R_{0}}}\left|\varphi_{+}(x)\right|^{2} d x,
\end{aligned}
$$

which gives $\varphi_{+} \equiv 0$, i.e. (a) holds.

Analogously, if $\tilde{f}(u(r))>v(r)$, we consider the test function

$$
\varphi_{-}(x):= \begin{cases}0 & \text { if } R_{0}<|x| \leq r \\ (v(|x|)-v(r))^{-} & \text {otherwise }\end{cases}
$$

and we prove that $(b)$ holds. Therefore, we have proved that $v$ is nondecreasing.

Case $u \in \mathcal{C}_{-, *}$. In this case, we know that $u \in \mathcal{C}_{-, *}$ and have to prove that $v$ is non-increasing. The proof is the same as for $\mathcal{C}_{+, *}$ changing the roles of $\varphi_{+}$and $\varphi_{-}$. 
It remains to show that $u_{-} \leq v \leq u_{+}$. By the fact that $\tilde{f}\left(u_{-}\right)=u_{-}$and that $\tilde{f}$ is nondecreasing we get

$$
(-\Delta)^{s}\left(v-u_{-}\right)+\left(v-u_{-}\right)=\tilde{f}(u)-\tilde{f}\left(u_{-}\right) \geq 0 .
$$

Multiplying the equation above by $\left(v-u_{-}\right)^{-}$, integrating it over $\Omega$, and using that for any $g$ one has that $-\left|g^{-}(x)-g^{-}(y)\right|^{2} \geq(g(x)-g(y))\left(g^{-}(x)-g^{-}(y)\right)$, we get

$$
\iint_{\mathbb{R}^{2 n} \backslash\left(\Omega^{c}\right)^{2}} \frac{\left|\left(v-u_{-}\right)^{-}(x)-\left(v-u_{-}\right)^{-}(y)\right|^{2}}{|x-y|^{n+2 s}} d x d y+\int_{\Omega}\left(v-u_{-}\right)\left(v-u_{-}\right)^{-} d x \leq 0,
$$

that is $\left(v-u_{-}\right)^{-} \equiv 0$ in $\Omega$. In a similar way, we prove that $v \leq u^{+}$in $\Omega\left(\right.$ if $\left.u^{+}<+\infty\right)$.

Remark 4.7. In what follows, we will use indifferently the quantities $\mathcal{E}^{\prime}(u), \nabla \mathcal{E}(u)$ and $u-\tilde{T}(u)$. Below, we write explicitly the relations among these three objects. Given $\mathcal{E}^{\prime}: H_{\Omega, 0}^{s} \rightarrow\left(H_{\Omega, 0}^{s}\right)^{*}$, the differential of $\mathcal{E}$, for every $u \in H_{\Omega, 0}^{s}$, we denote by $\nabla \mathcal{E}(u)$ the only function of $H_{\Omega, 0}^{s}$ (whose existence is guaranteed by Riesz's Representation Theorem) such that

$$
(\nabla \mathcal{E}(u), v)_{H_{\Omega, 0}^{s}}=\mathcal{E}^{\prime}(u)[v] \quad \text { for all } v \in H_{\Omega, 0}^{s},
$$

where $(\cdot, \cdot)_{H_{\Omega, 0}^{s}}$ is the scalar product defined in Section 2. In particular, $\|\nabla \mathcal{E}(u)\|_{H_{\Omega, 0}^{s}}=\left\|\mathcal{E}^{\prime}(u)\right\|_{*}$, $\|\cdot\|_{*}$ being the norm in the dual space $\left(H_{\Omega, 0}^{s}\right)^{*}$. Now, by the definition (4.17) of the operator $\tilde{T}$, we know that, for every $u \in H_{\Omega, 0}^{s}, \tilde{T}(u)=v$, where $v \in H_{\Omega, 0}^{s}$ is the unique solution of $(-\Delta)^{s} v+$ $v=\tilde{f}(u)$ in $\Omega$, under nonlocal Neumann boundary conditions. Therefore, for every $u, v \in H_{\Omega, 0}^{s}$, it results

$$
\begin{aligned}
(u & -\tilde{T}(u), v)_{H_{\Omega, 0}^{s}}= \\
= & \frac{c_{n, s}}{2} \iint_{\mathbb{R}^{2 n} \backslash\left(\Omega^{c}\right)^{2}} \frac{(u(x)-u(y))(v(x)-v(y))}{|x-y|^{n+2 s}} d x d y+\int_{\Omega} u v d x \\
& -\frac{c_{n, s}}{2} \iint_{\mathbb{R}^{2 n} \backslash\left(\Omega^{c}\right)^{2}} \frac{(\tilde{T}(u(x))-\tilde{T}(u(y)))(v(x)-v(y))}{|x-y|^{n+2 s}} d x d y-\int_{\Omega} \tilde{T}(u) v d x \\
= & \frac{c_{n, s}}{2} \iint_{\mathbb{R}^{2 n} \backslash\left(\Omega^{c}\right)^{2}} \frac{(u(x)-u(y))(v(x)-v(y))}{|x-y|^{n+2 s}} d x d y+\int_{\Omega}(u-\tilde{f}(u)) v d x \\
= & \mathcal{E}^{\prime}(u)[v] .
\end{aligned}
$$

In conclusion, $u-\tilde{T}(u)=\nabla \mathcal{E}(u)$ for every $u \in H_{\Omega, 0}^{s}$.

Lemma 4.8 (Deformation Lemma in $\left.\mathcal{C}_{*}\right)$. Let $c \in \mathbb{R}$ be such that $\mathcal{E}^{\prime}(u) \neq 0$ for all $u \in \mathcal{C}_{*}$, with $\mathcal{E}(u)=c$. Then, there exists a function $\eta: \mathcal{C}_{*} \rightarrow \mathcal{C}_{*}$ satisfying the following properties: 
(i) $\eta$ is continuous with respect to the topology of $H_{\Omega, 0}^{s}$;

(ii) $\mathcal{E}(\eta(u)) \leq \mathcal{E}(u)$ for all $u \in \mathcal{C}_{*}$;

(iii) $\mathcal{E}(\eta(u)) \leq c-\bar{\varepsilon}$ for all $u \in \mathcal{C}_{*}$ such that $|\mathcal{E}(u)-c|<\bar{\varepsilon}$;

(iv) $\eta(u)=u$ for all $u \in \mathcal{C}_{*}$ such that $|\mathcal{E}(u)-c|>2 \bar{\varepsilon}$,

where $\bar{\varepsilon}$ is the positive constant corresponding to c given in Corollary 4.4.

Proof. The ideas of this proof are borrowed from [4, Lemma 4.5], cf. also [8, Lemma 3.8]. Let $\chi_{1}: \mathbb{R} \rightarrow[0,1]$ be a smooth cut-off function such that

$$
\chi_{1}(t)= \begin{cases}1 & \text { if }|t-c|<\bar{\varepsilon} \\ 0 & \text { if }|t-c|>2 \bar{\varepsilon}\end{cases}
$$

where $\bar{\delta}$ and $\bar{\varepsilon}$ are given in Corollary 4.4. Let $\Phi: H_{\Omega, 0}^{s} \rightarrow H_{\Omega, 0}^{s}$ be the map defined by

$$
\Phi(u):= \begin{cases}\chi_{1}(\mathcal{E}(u)) \frac{\nabla \mathcal{E}(u)}{\|\nabla \mathcal{E}(u)\|_{H_{\Omega, 0}^{s}}} & \text { if }|\mathcal{E}(u)-c| \leq 2 \bar{\varepsilon} \\ 0 & \text { otherwise. }\end{cases}
$$

Note that the definition of $\Phi$ is well posed by Corollary 4.4.

For all $u \in \mathcal{C}_{*}$, we consider the Cauchy problem

$$
\left\{\begin{array}{l}
\frac{d}{d t} \eta(t, u)=-\Phi(\eta(t, u)) \quad t \in(0, \infty) \\
\eta(0, u)=u
\end{array}\right.
$$

Being $\mathcal{E}$ of class $C^{2}$, there exists a unique solution $\eta(\cdot, u) \in C^{1}\left([0, \infty) ; H_{\Omega, 0}^{s}\right)$, cf. [10, Chapter \$1].

We shall prove that for all $t>0, \eta\left(t, \mathcal{C}_{*}\right) \subset \mathcal{C}_{*}$. Fix $\bar{t}>0$. For every $u \in \mathcal{C}_{*}$ and $k \in \mathbb{N}$ with $k \geq \bar{t} / \bar{\delta}$, let

$$
\left\{\begin{array}{l}
\bar{\eta}_{k}(0, u):=u, \\
\bar{\eta}_{k}\left(t_{i+1}, u\right):=\bar{\eta}_{k}\left(t_{i}, u\right)-\frac{\bar{t}}{k} \Phi\left(\bar{\eta}_{k}\left(t_{i}, u\right)\right) \quad \text { for all } i=0, \ldots, k-1,
\end{array}\right.
$$

with

$$
t_{i}:=i \cdot \frac{\bar{t}}{k} \quad \text { for all } i=0, \ldots, k-1 .
$$

Let us prove that for all $i=0, \ldots, k-1, \bar{\eta}_{k}\left(t_{i+1}, u\right) \in \mathcal{C}_{*}$. If $|\mathcal{E}(u)-c|>2 \bar{\varepsilon}$, then $\bar{\eta}_{k}\left(t_{i+1}, u\right)=$ $u \in \mathcal{C}_{*}$ for every $i=0, \ldots, k-1$. Otherwise, let

$$
\lambda:=\frac{\bar{t}}{k} \cdot \frac{\chi_{1}\left(\mathcal{E}\left(\bar{\eta}_{k}\left(t_{i}, u\right)\right)\right)}{\left\|\bar{\eta}_{k}\left(t_{i}, u\right)-\tilde{T}\left(\bar{\eta}_{k}\left(t_{i}, u\right)\right)\right\|_{H_{\Omega, 0}^{s}}} .
$$

Clearly, $\lambda \leq 1$ by Corollary 4.4 , being $k \geq \bar{t} / \bar{\delta}$ and $\|u-\tilde{T}(u)\|_{H_{\Omega, 0}^{s}}=\|\nabla \mathcal{E}\|_{H_{\Omega, 0}^{s}}$. Therefore, we have for every $i=0, \ldots, k-1$ 


$$
\bar{\eta}_{k}\left(t_{i+1}, u\right)=(1-\lambda) \bar{\eta}_{k}\left(t_{i}, u\right)+\lambda \tilde{T}\left(\bar{\eta}_{k}\left(t_{i}, u\right)\right) \in \mathcal{C}_{*}
$$

by induction on $i$, and by the convexity of $\mathcal{C}_{*}$. For every $i=0, \ldots, k-1$, we can now define the 3

line segment

$$
\eta_{k}^{(i)}(t, u):=\left(1-\frac{t}{\bar{t}} k+i\right) \bar{\eta}_{k}\left(t_{i}, u\right)+\left(\begin{array}{l}
t \\
\overline{\bar{t}}
\end{array}-i\right) \bar{\eta}_{k}\left(t_{i+1}, u\right)
$$

for all $t \in\left[t_{i}, t_{i+1}\right]$. We denote by $\eta_{k}:=\bigcup_{i=0}^{k-1} \eta_{k}^{(i)}$ the whole Euler polygonal defined in $[0, \bar{t}]$. Being $\mathcal{C}_{*}$ convex, we get immediately that for all $t \in[0, \bar{t}], \eta_{k}(t, u) \in \mathcal{C}_{*}$.

We claim that $\eta_{k}(\cdot, u)$ converges to the solution $\eta(\cdot, u)$ of the Cauchy problem $(4.20)$ in $H_{\Omega, 0}^{s}$. Indeed, for all $i=0, \ldots, k-1$, we integrate by parts the equation of (4.20) in the interval $\left[t_{i}, t_{i+1}\right]$ and we obtain

$$
\eta\left(t_{i+1}, u\right)=\eta\left(t_{i}, u\right)-\frac{\bar{t}}{k} \Phi\left(\eta\left(t_{i}, u\right)\right)+\int_{t_{i}}^{t_{i+1}}\left(\tau-t_{i+1}\right) \frac{d}{d \tau} \Phi(\eta(\tau, u)) d \tau .
$$

On the other hand, we define the error

$$
\varepsilon_{i}:=\left\|\eta\left(t_{i}, u\right)-\eta_{k}\left(t_{i}, u\right)\right\|_{H_{\Omega, 0}^{s}} \quad \text { for every } i=0, \ldots, k-1 .
$$

Hence, for every $i=0, \ldots, k-1$, we get

$$
\begin{aligned}
\varepsilon_{i+1} \leq \varepsilon_{i}+\frac{\bar{t}}{k} & \left\|\Phi\left(\eta\left(t_{i}, u\right)\right)-\Phi\left(\eta_{k}\left(t_{i}, u\right)\right)\right\|_{H_{\Omega, 0}^{s}} \\
& +\left\|\int_{t_{i}}^{t_{i+1}}\left(t_{i+1}-\tau\right) \frac{d}{d \tau} \Phi(\eta(\tau, u)) d \tau\right\|_{H_{\Omega, 0}^{s}} .
\end{aligned}
$$

Now, since $\Phi$ is locally Lipschitz and $\eta([0, \bar{t}]) \subset H_{\Omega, 0}^{s}$ is compact,

$$
\left\|\Phi\left(\eta\left(t_{i}, u\right)\right)-\Phi\left(\eta_{k}\left(t_{i}, u\right)\right)\right\|_{H_{\Omega, 0}^{s}} \leq \varepsilon_{i} L_{\Phi}
$$

for some $L_{\Phi}=L_{\Phi}(\eta([0, \bar{t}]))>0$. Furthermore,

$$
\begin{aligned}
\left\|\int_{t_{i}}^{t_{i+1}}\left(t_{i+1}-\tau\right) \frac{d}{d \tau} \Phi(\eta(\tau, u)) d \tau\right\|_{H_{\Omega, 0}^{s}} & \leq \int_{t_{i}}^{t_{i+1}}\left(t_{i+1}-\tau\right)\left\|\frac{d}{d \tau} \Phi(\eta(\tau, u))\right\|_{H_{\Omega, 0}^{s}} d \tau \\
& \leq \frac{\bar{t}}{k} \int_{0}^{\bar{t}}\left\|\Phi^{\prime}(\eta(\tau, u))\right\|_{*}\|\Phi(\eta(\tau, u))\|_{H_{\Omega, 0}^{s}} d \tau \\
& \leq \frac{\bar{t}^{2}}{k} \sup _{\tau \in[0, \bar{t}]}\left\|\Phi^{\prime}(\eta(\tau, u))\right\|_{*}=\frac{\bar{t}^{2}}{k} L_{\Phi} .
\end{aligned}
$$


Thus, combining the last inequality with (4.22) and (4.21), we have

$$
\varepsilon_{i+1} \leq \varepsilon_{i}+\frac{\bar{t}}{k} \varepsilon_{i} L_{\Phi}+\frac{\bar{t}^{2}}{k} L_{\Phi} \quad \text { for all } i=0, \ldots, k-1 .
$$

This implies that

$$
\varepsilon_{i+1} \leq \frac{\bar{t}^{2}}{k} L_{\Phi} \sum_{j=0}^{i}\left(1+\frac{\bar{t}}{k} L_{\Phi}\right)^{j}=\bar{t}\left[\left(1+\frac{\bar{t}}{k} L_{\Phi}\right)^{i+1}-1\right] \rightarrow 0 \quad \text { as } k \rightarrow \infty,
$$

where we have used the fact that $\varepsilon_{0}=0$. By the triangle inequality and the continuity of $\eta(\cdot, u)$ and $\eta_{k}(\cdot, u)$, this yields the claim.

Hence, for all $t \in[0, \bar{t}], \eta(t, u) \in \mathcal{C}_{*}$ by the closedness of $\mathcal{C}_{*}$.

For all $u \in \mathcal{C}_{*}$ and $t>0$ we can write

$$
\begin{aligned}
& \mathcal{E}(\eta(t, u))-\mathcal{E}(u)=\int_{0}^{t} \frac{d}{d \tau} \mathcal{E}(\eta(\tau, u)) d \tau \\
& =-\int_{0}^{t} \frac{\chi_{1}(\mathcal{E}(\eta(\tau, u)))}{\|\eta(\tau, u)-\tilde{T}(\eta(\tau, u))\|_{H_{\Omega, 0}^{s}}} \mathcal{E}^{\prime}(\eta(\tau, u))[\eta(\tau, u)-\tilde{T}(\eta(\tau, u))] d \tau \\
& =-\int_{0}^{t}\|\eta(\tau, u)-\tilde{T}(\eta(\tau, u))\|_{H_{\Omega, 0}^{s}} \chi_{1}(\mathcal{E}(\eta(\tau, u))) d \tau \leq 0 .
\end{aligned}
$$

Now, let $u \in \mathcal{C}_{*}$ be such that $|\mathcal{E}(u)-c|<\bar{\varepsilon}$ and let $t \geq 2 \bar{\varepsilon} / \bar{\delta}$. Then, two cases arise: either there exists $\tau \in[0, t]$ for which $\mathcal{E}(\eta(\tau, u)) \leq c-\bar{\varepsilon}$ and so, by the previous calculation we get immediately that $\mathcal{E}(\eta(t, u)) \leq c-\bar{\varepsilon}$, or for all $\tau \in[0, t], \mathcal{E}(\eta(\tau, u))>c-\bar{\varepsilon}$. In this second case,

$$
c-\bar{\varepsilon}<\mathcal{E}(\eta(\tau, u)) \leq \mathcal{E}(u)<c+\bar{\varepsilon} .
$$

In particular, by the definition of $\chi_{1}$, and by Corollary 4.4 , we have that for all $\tau \in[0, t]$

$$
\chi_{1}(\mathcal{E}(\eta(\tau, u)))=1, \quad\|\eta(\tau, u)-\tilde{T}(\eta(\tau, u))\|_{H_{\Omega, 0}^{s}} \geq \bar{\delta} .
$$

Hence, by (4.23), we obtain

$$
\mathcal{E}(\eta(t, u)) \leq \mathcal{E}(u)-\int_{0}^{t} \bar{\delta} d \tau \leq c+\bar{\varepsilon}-\bar{\delta} t \leq c-\bar{\varepsilon} .
$$

Finally, if we define with abuse of notation

$$
\eta(u):=\eta\left(\frac{2 \bar{\varepsilon}}{\bar{\delta}}, u\right)
$$

it is immediate to verify that $\eta$ satisfies (i)-(iv). 
Lemma 4.9 (Mountain pass geometry). Let $\tau>0$ be such that $\tau<\min \left\{u_{0}-u_{-}, u_{+}-u_{0}\right\} . \quad 1$

Then there exists $\alpha>0$ such that

(i) $\mathcal{E}(u) \geq \mathcal{E}\left(u_{-}\right)+\alpha$ for every $u \in \mathcal{C}_{*}$ with $\left\|u-u_{-}\right\|_{L^{\infty}(\Omega)}=\tau$;

(ii) if $u_{+}<\infty$, then $\mathcal{E}(u) \geq \mathcal{E}\left(u_{+}\right)+\alpha$ for every $u \in \mathcal{C}_{*}$ with $\left\|u-u_{+}\right\|_{L^{\infty}(\Omega)}=\tau$;

(iii) if $u_{+}=+\infty$, then there exists $\bar{u} \in \mathcal{C}_{*}$ with $\left\|\bar{u}-u_{-}\right\|_{L^{\infty}(\Omega)}>\tau$ such that $\mathcal{E}(\bar{u})<\mathcal{E}\left(u_{-}\right)$.

Proof. The proof is analogous to the one of [4, Lemma 4.6], we report it here for the sake of 8 completeness. Suppose by contradiction that there exists a sequence $\left(w_{k}\right) \subset \mathcal{C}_{*}$ such that

$$
\left\|w_{k}\right\|_{L^{\infty}(\Omega)}=w_{k}(R)=\tau>0 \quad \text { for all } k
$$

$$
\begin{aligned}
& \text { and } \limsup _{k \rightarrow \infty}\left[\mathcal{E}\left(u_{-}+w_{k}\right)-\mathcal{E}\left(u_{-}\right)\right] \leq 0 \text {. Since } \\
& \qquad \begin{array}{l}
\frac{1}{2} \int_{\Omega}\left(\left(u_{-}+w_{k}\right)^{2}-u_{-}^{2}\right) d x=\int_{\Omega} \int_{0}^{1}\left(u_{-}+t w_{k}\right) w_{k} d t d x, \\
\tilde{F}\left(u_{-}+w_{k}\right)-\tilde{F}\left(u_{-}\right)=\int_{0}^{1} \tilde{f}\left(u_{-}+t w_{k}\right) w_{k} d t
\end{array}
\end{aligned}
$$

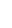

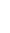

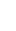

we get

$$
\begin{aligned}
\mathcal{E} & \left(u_{-}+w_{k}\right)-\mathcal{E}\left(u_{-}\right) \\
& =\frac{1}{2}\left(\left[w_{k}\right]_{H_{\Omega, 0}^{s}}^{2}+\int_{\Omega}\left[\left(u_{-}+w_{k}\right)^{2}-u_{-}^{2}\right] d x\right)-\int_{\Omega}\left(\tilde{F}\left(u_{-}+w_{k}\right)-\tilde{F}\left(u_{-}\right)\right) d x \\
& =\frac{1}{2}\left(\left[w_{k}\right]_{H_{\Omega, 0}^{s}}^{2}+\int_{\Omega} \int_{0}^{1}\left(u_{-}+t w_{k}-\tilde{f}\left(u_{-}+t w_{k}\right)\right) w_{k} d t d x\right) .
\end{aligned}
$$

Therefore, since by $\left(f_{3}\right)$ and the definition of $u_{-}$

$$
t-\tilde{f}(t)>0 \quad \text { for } t \in\left(u_{-}, u_{0}\right)
$$

we conclude that $\left[w_{k}\right]_{H_{\Omega, 0}^{s}} \rightarrow 0$. We claim that $\left(w_{k}\right)$ converges to the constant solution $w \equiv \tau$ in the $H_{\Omega, 0}^{s}$ norm. Indeed, using $\left[w_{k}\right]_{H_{\Omega, 0}^{s}} \rightarrow 0$ and (4.24), we have that $\left(w_{k}\right)$ is bounded in $H_{\Omega, 0}^{s}$ and so, up to a subsequence, it weakly converges to some $w \in H_{\Omega, 0}^{s}$. Hence,

$$
\begin{aligned}
0 & =\lim _{k \rightarrow \infty} \iint_{\mathbb{R}^{2 n} \backslash\left(\Omega^{c}\right)^{2}} \frac{\left[\left(w_{k}-w\right)(x)-\left(w_{k}-w\right)(y)\right](w(x)-w(y))}{|x-y|^{n+2 s}} d x d y \\
& =\lim _{k \rightarrow \infty} \iint_{\mathbb{R}^{2 n} \backslash\left(\Omega^{c}\right)^{2}} \frac{\left(w_{k}(x)-w_{k}(y)\right)(w(x)-w(y))}{|x-y|^{n+2 s}} d x d y-[w]_{H_{\Omega, 0}^{s}}^{2} .
\end{aligned}
$$


Moreover,

$$
\frac{c_{n, s}}{2} \iint_{\mathbb{R}^{2 n} \backslash\left(\Omega^{c}\right)^{2}} \frac{\left(w_{k}(x)-w_{k}(y)\right)(w(x)-w(y))}{|x-y|^{n+2 s}} d x d y \leq C\left[w_{k}\right]_{H_{\Omega, 0}^{s}}[w]_{H_{\Omega, 0}^{s}} .
$$

Combining (4.26) and (4.27), we get $[w]_{H_{\Omega, 0}^{s}}=0$, which implies that $w \equiv \tau$. Thus, $\left(w_{k}\right)$ converges to the constant $\tau$ in $H_{\Omega, 0}^{s}$. By the Dominated Convergence Theorem we can conclude that

$$
\begin{aligned}
0 & \geq \lim _{k \rightarrow \infty} \int_{\Omega} \int_{0}^{1}\left(u_{-}+t w_{k}-\tilde{f}\left(u_{-}+t w_{k}\right)\right) w_{k} d t d x \\
& =\int_{\Omega} \int_{0}^{1}\left(u_{-}+t \tau-\tilde{f}\left(u_{-}+t \tau\right)\right) \tau d t d x,
\end{aligned}
$$

which contradicts (4.25). Hence there exists $\alpha_{1}>0$ such that (i) holds.

In a similar way, now using the fact that $t-\tilde{f}(t)<0$ for $t \in\left(u_{0}, u_{+}\right)$, we find $\alpha_{2}>0$ such that (ii) holds if $u_{+}<\infty$. The claim then follows with $\alpha:=\min \left\{\alpha_{1}, \alpha_{2}\right\}$.

Finally, if $u_{+}=+\infty$, the existence of a point $\bar{u} \in \mathcal{C}_{*}$ outside the crest centered in $u_{-}$is guaranteed by the following estimate (cf. also [7, Remarks p. 118]):

$$
\begin{aligned}
\mathcal{E}(t \cdot 1) & =|\Omega|\left(\frac{t^{2}}{2}-\int_{0}^{t} \tilde{f}(s) d s\right) \\
& \leq|\Omega|\left(\frac{t^{2}}{2}-\int_{0}^{M} \tilde{f}(s) d s-(1+\delta) \int_{M}^{t} s d s\right) \\
& \leq \frac{|\Omega|}{2}\left(t^{2}-2 M \min _{s \in[0, M]} \tilde{f}(s)-(1+\delta)\left(t^{2}-M^{2}\right)\right) \\
& =C-\frac{|\Omega| \delta}{2} t^{2} \rightarrow-\infty \quad \text { as } t \rightarrow \infty,
\end{aligned}
$$

where we have used the fact that $\tilde{f} \in \mathfrak{F}_{M, \delta}$. This shows (iii) and concludes the proof.

Remark 4.10. We observe that, comparing (i) and (ii) in Lemma 4.9, it is apparent that, whenever $u_{+}<+\infty$, if $\mathcal{E}\left(u_{-}\right)<\mathcal{E}\left(u_{+}\right)$, then $u_{+}$plays the role of the center inside the crest of the mountain pass and $u_{-}$plays the role of the point outside the crest with less energy, otherwise the roles of $u_{-}$and $u_{+}$have to be interchanged. 
Now, let

$$
\begin{aligned}
& U_{-}:=\left\{u \in \mathcal{C}_{*}: \mathcal{E}(u)<\mathcal{E}\left(u_{-}\right)+\frac{\alpha}{2},\left\|u-u_{-}\right\|_{L^{\infty}(\Omega)}<\tau\right\}, \\
& U_{+}:= \begin{cases}\left\{u \in \mathcal{C}_{*}: \mathcal{E}(u)<\mathcal{E}\left(u_{+}\right)+\frac{\alpha}{2},\left\|u-u_{+}\right\|_{L^{\infty}(\Omega)}<\tau\right\}, & \text { if } u_{+}<\infty, \\
\left\{u \in \mathcal{C}_{*}: \mathcal{E}(u)<\mathcal{E}\left(u_{-}\right),\left\|u-u_{-}\right\|_{L^{\infty}(\Omega)}>\tau\right\}, & \text { if } u_{+}=\infty\end{cases}
\end{aligned}
$$

where $\tau$ and $\alpha$ are given by Lemma 4.9,

$$
\Gamma:=\left\{\gamma \in C\left([0,1] ; \mathcal{C}_{*}\right): \gamma(0) \in U_{-}, \gamma(1) \in U_{+}\right\},
$$

and

$$
c:=\inf _{\gamma \in \Gamma} \max _{t \in[0,1]} \mathcal{E}(\gamma(t))
$$

Remark 4.11. The reason for considering two sets, $U_{+}$and $U_{-}$, instead of just two points for the starting and the ending points of the admissible curves will be clear in Lemma 5.3. Indeed, this choice makes easier exhibiting an admissible curve along which the energy is lower than the energy of the constant.

Proposition 4.12 (Mountain Pass Theorem). The value $c$ defined in (4.30) is finite and there exists a critical point $u \in \mathcal{C}_{*} \backslash\left\{u_{-}, u_{+}\right\}$of $\mathcal{E}$ with $\mathcal{E}(u)=c$. In particular, $u$ is a weak solution of (1.1).

The proof of the above proposition is standard, once one has the mountain pass geometry (Lemma 4.9) and the deformation Lemma (Lemma 4.8). We refer e.g. to [8, Proposition 3.10] for a proof given in a very similar situation.

\section{Non-constancy of the minimax solution}

In this section we prove that the solution $u \in \mathcal{C}_{*}$, whose existence has been established in the previous section, is non-constant. Since we work in the restricted cone $\mathcal{C}_{*}$ where the only constant solutions are $u_{-}, u_{+}$, and $u_{0}$, and since the mountain pass geometry guarantees that $u \neq u_{-}$and $u \not \equiv u_{+}$(cf. Proposition 4.12), it is enough to prove that $u \not \equiv u_{0}$. To this aim, following the idea in $[4$, Section 4$]$, we first prove that on the Nehari-type set

$$
N_{*}:=\left\{u \in \mathcal{C}_{*} \backslash\{0\}: \mathcal{E}^{\prime}(u)[u]=0\right\},
$$

i.e., roughly speaking, on the crest of the mountain pass, the infimum of the energy is strictly less than $\mathcal{E}\left(u_{0}\right)$, cf. also [8, Remark 2]. Then, we explicitly build an admissible curve $\bar{\gamma} \in \Gamma$ along which the energy is less than $\mathcal{E}\left(u_{0}\right)$. By (4.30), this ensures that the mountain pass level is less than $\mathcal{E}\left(u_{0}\right)$ and so $u \not \equiv u_{0}$. 
We start by introducing some useful notation. We denote by

$$
H_{\mathrm{rad}}^{s}:=\left\{u \in H_{\Omega, 0}^{S}: u \text { radial }\right\},
$$

we introduce also the space of radial, non-decreasing functions

$$
H_{+, \mathrm{r}}^{s}:=\left\{u \in H_{\Omega, 0}^{s}: u \text { radial and radially non-decreasing }\right\} .
$$

$$
\lambda_{2}^{\mathrm{rad}}:=\inf _{v \in H_{\mathrm{rad}}^{s}, \int v=0} \frac{[v]_{H_{\Omega, 0}^{s}}^{2}}{\int_{\Omega} v^{2}}, \quad \lambda_{2}^{+, \mathrm{r}}:=\inf _{v \in H_{+, \mathrm{r}}^{s}, \int v=0} \frac{[v]_{H_{\Omega, 0}^{s}}^{2}}{\int_{\Omega} v^{2}} .
$$

Clearly, the following chain of inequalities holds by inclusion $H_{+, \mathrm{r}}^{s} \subset H_{\text {rad }}^{s} \subset H_{\Omega, 0}^{s}$

$$
0<\lambda_{2} \leq \lambda_{2}^{\mathrm{rad}} \leq \lambda_{2}^{+, \mathrm{r}}
$$

and, by the direct method of Calculus of Variations, all these infima are achieved.

Remark 5.1. We observe that in the local case, i.e., for the Neumann Laplacian, it is known that the second radial eigenfunction is increasing, so that the second radial eigenvalue and the second radial increasing eigenvalue coincide. In this nonlocal setting we do not know whether the same equality holds true. In [4], for the local case, the condition required on $f^{\prime}\left(u_{0}\right)$ involves the second radial eigenvalue, and the proof of the non-constancy of the solution uses the monotonicity of the associated eigenfunction. In this paper, we need to require an assumption involving $\lambda_{2}^{+, r}$, which, as explained above, might be more restrictive. On the other hand, as will be clear in Proposition 5.4, some condition on the derivative of $f$ is needed in order to guarantee the existence of non-constant solutions.

Lemma 5.2. Let $v_{2} \in H_{+, \mathrm{r}}^{s}$ be the second radial increasing eigenfunction, namely the function that realizes $\lambda_{2}^{+, \mathrm{r}}$. Let

$$
\psi: \mathbb{R}^{2} \rightarrow \mathbb{R}, \quad \psi(s, t):=\mathcal{E}^{\prime}\left(t\left(u_{0}+s v_{2}\right)\right)\left[u_{0}+s v_{2}\right]
$$

then there exist $\varepsilon_{1}, \varepsilon_{2}>0$ and $a C^{1}$ function $h:\left(-\varepsilon_{1}, \varepsilon_{1}\right) \rightarrow\left(1-\varepsilon_{2}, 1+\varepsilon_{2}\right)$ such that for $(s, t) \in V:=\left(-\varepsilon_{1}, \varepsilon_{1}\right) \times\left(1-\varepsilon_{2}, 1+\varepsilon_{2}\right)$ we have

$$
\psi(s, t)=0 \text { if and only if } t=h(s) .
$$

\section{Moreover,}

(i) $h(0)=1, h^{\prime}(0)=0$;

(ii) $\frac{\partial}{\partial t} \psi(s, t)<0$ for $(s, t) \in V$;

(iii) $\mathcal{E}\left(h(s)\left(u_{0}+s v_{2}\right)\right)<\mathcal{E}\left(u_{0}\right)$ for $s \in\left(-\varepsilon_{1}, \varepsilon_{1}\right), s \neq 0$. 
The same result holds true replacing $v_{2}$ with the second radial decreasing eigenfunction $-v_{2}$ (which clearly corresponds to the same eigenvalue $\lambda_{2}^{+, \mathrm{r}}$ ).

Proof. The proof is similar to the one of [4, Lemma 4.9], we report it here because it highlights the importance of assumption $\left(f_{3}\right)$. Part (i) follows by the Implicit Function Theorem applied to $\psi$. Indeed, since $\mathcal{E}$ is a $C^{2}$ functional and $\psi$ is of class $C^{1}$ with $\psi(0,1)=0$, by $\left(f_{3}\right)$ we get

$$
\left.\frac{\partial}{\partial t}\right|_{(0,1)} \psi(s, t)=\mathcal{E}^{\prime \prime}\left(u_{0}\right)\left[u_{0}, u_{0}\right]=\left[1-\tilde{f}^{\prime}\left(u_{0}\right)\right] \int_{B} u_{0}^{2} d x<0
$$

where we have used only that $\tilde{f}^{\prime}\left(u_{0}\right)=f^{\prime}\left(u_{0}\right)>1$. Furthermore, since $\int_{\Omega} v_{2}=0$,

$$
\begin{aligned}
\left.\frac{\partial}{\partial s}\right|_{(0,1)} \psi(s, t) & =\mathcal{E}^{\prime}\left(u_{0}\right)\left[v_{2}\right]+\mathcal{E}^{\prime \prime}\left(u_{0}\right)\left[u_{0}, v_{2}\right] \\
& =\left[1-\tilde{f}^{\prime}\left(u_{0}\right)\right] u_{0} \int_{\Omega} v_{2} d x=0 .
\end{aligned}
$$

Thus, the Implicit Function Theorem guarantees the existence of $\varepsilon_{1}, \varepsilon_{2}$ and $h$, as well as property (i). Then, part (ii) is a consequence of the regularity of $\psi$. We prove now (iii), here is where $\left(f_{3}\right)$ plays a crucial role. By (i), we can write $h(s)=1+o(s)$, for $s \in\left(-\varepsilon_{1}, \varepsilon_{1}\right), s \neq 0$, so that

$$
h(s)\left(u_{0}+s v_{2}\right)-u_{0}=s v_{2}+o(s)
$$

and therefore, by Taylor expansion and $\left(f_{3}\right)$,

$$
\begin{aligned}
\mathcal{E}\left(h(s)\left(u_{0}+s v_{2}\right)\right)-\mathcal{E}\left(u_{0}\right) & =\frac{1}{2} \mathcal{E}^{\prime \prime}\left(u_{0}\right)\left[s v_{2}+o(s), s v_{2}+o(s)\right]+o\left(s^{2}\right) \\
& =\frac{s^{2}}{2} \mathcal{E}^{\prime \prime}\left(u_{0}\right)\left[v_{2}, v_{2}\right]+o\left(s^{2}\right) \\
& =\frac{s^{2}}{2}\left(\left[v_{2}\right]_{H_{\Omega, 0}^{s}}^{2}+\int_{\Omega}\left[1-\tilde{f}^{\prime}\left(u_{0}\right)\right] v_{2}^{2} d x\right)+o\left(s^{2}\right) \\
& <\frac{s^{2}}{2}\left(\left[v_{2}\right]_{H_{\Omega, 0}^{s}}^{2}-\lambda_{2}^{+, \mathrm{r}} \int_{\Omega} v_{2}^{2} d x\right)+o\left(s^{2}\right) .
\end{aligned}
$$

Then, being

property (iii) holds taking $\varepsilon_{1}, \varepsilon_{2}$ smaller if necessary. 
In the following lemma, we build a curve $\gamma_{\bar{\tau}}$ along which the energy is always less than $\mathcal{E}\left(u_{0}\right)$. The admissible curve $\bar{\gamma} \in \Gamma$ with the same property will be a simple reparametrization of $\gamma_{\bar{\tau}}$.

Lemma 5.3. Fix $0<t_{-}<1<t_{+}$such that

$$
t_{-} u_{0} \in U_{-}, \quad t_{+} u_{0} \in U_{+} \quad \text { and } \quad u_{-}<t_{-} u_{0}<u_{0}<t_{+} u_{0}<u_{+},
$$

where $U_{ \pm}$are defined in (4.29). Let $v_{2}$ be the second radial increasing eigenfunction as in Lemma 5.2. For $\tau \geq 0$ define

$$
\begin{array}{ll}
\gamma_{\tau}:\left[t_{-}, t_{+}\right] \rightarrow H_{\Omega, 0}^{s} & \gamma_{\tau}(t):=t\left(u_{0}+\tau v_{2}\right) \\
& \left(\text { resp. } \gamma_{\tau}(t):=t\left(u_{0}-\tau v_{2}\right)\right) .
\end{array}
$$

Then there exists $\bar{\tau}>0$ such that $\gamma_{\bar{\tau}}\left(t_{ \pm}\right) \in U_{ \pm}, \gamma_{\bar{\tau}}(t) \in \mathcal{C}_{+, *}\left(\right.$ resp. $\left.\mathcal{C}_{-, *}\right)$ for $t_{-} \leq t \leq t_{+}$and

$$
\max _{t_{-} \leq t \leq t_{+}} \mathcal{E}\left(\gamma_{\bar{\tau}}(t)\right)<\mathcal{E}\left(u_{0}\right)
$$

As a consequence, there exists an admissible curve $\bar{\gamma} \in \Gamma$ along which the energy is always lower than $\mathcal{E}\left(u_{0}\right)$.

For the proof of the previous lemma, we refer to [4, Lemma 4.10], see also [8, Lemma 4.2]. Here the monotonicity of $v_{2}$ (resp. of $\left.-v_{2}\right)$ is essential to guarantee that $\gamma_{\bar{\tau}}\left(\left[t_{-}, t_{+}\right]\right) \subset \mathcal{C}_{+, *}$ (resp. $\left.\mathcal{C}_{-, *}\right)$. Finally, the admissible curve $\gamma \in \Gamma$ is given in terms of $\gamma_{\bar{\tau}}$ as follows

$$
\bar{\gamma}(t):=\gamma_{\bar{\tau}}\left(t\left(t_{+}-t_{-}\right)+t_{-}\right) \quad \text { for all } t \in[0,1]
$$

1

- Proof of Theorem 1.1. By Proposition 4.12, there exists a mountain pass type solution $u \in$ $\mathcal{C}_{*} \backslash\left\{u_{-}, u_{+}\right\}$of $(1.1)$ such that $\mathcal{E}(u)=c$. Moreover, by Lemma 5.3 and the definition of the minimax level $c$ given in (4.30), we have that

$$
c \leq \max _{t \in[0,1]} \mathcal{E}(\bar{\gamma}(t))<\mathcal{E}\left(u_{0}\right)
$$

that is $u \not \equiv u_{0}$, and so $u$ is non-constant. Furthermore, $u>0$ a.e. in $\Omega$ by the maximum principle stated in Theorem 2.6 combined with the regularity of $u$ given in Lemma 3.6. Actually, since $u$ is smooth and non-decreasing, $u>0$ in $\Omega \backslash\{0\}$.

The multiplicity part of the statement is proved by reasoning in the same way for each $u_{0, i}$, with $i=1, \ldots, N$. Indeed, assume without loss of generality that $u_{0,1}<u_{0,2}<\cdots<u_{0, N}$. For every $i$, we define $u_{ \pm, i}$ and the cone of non-negative, radial, non-decreasing (or non-increasing) functions $\mathcal{C}_{*, i}$, corresponding to $u_{0, i}$. Then

$$
u_{-, 1}<u_{+, 1} \leq u_{-, 2}<\cdots \leq u_{+, N}
$$

Proceeding as in the present and in the previous sections, for every $i$, we get a non-constant positive solution $u_{i} \in \mathcal{C}_{*, i}$. Hence, by (5.8), 


$$
u_{-, 1} \underset{\not \equiv}{\leq} u_{1} \leq u_{+, 1} \leq u_{-, 2} \underset{\not \equiv}{\leq} u_{2} \underset{\not \equiv}{\leq} u_{+, 2} \underset{\not \equiv}{\leq} \cdots \underset{\not \equiv}{\leq} u_{N} \leq u_{+, N},
$$

which proves in particular that the $N$ solutions are distinct.

The following proposition gives a sufficient condition on $f$ under which problem (1.1) admits only constant solutions. We recall that $K_{\infty}$ denotes the uniform bound on the $L^{\infty}$ norm of $u$ given in Lemma 3.5.

Proposition 5.4. Let $\delta \in\left(0, \lambda_{2}^{\mathrm{rad}}\right)$ and $M>0$. Suppose that $f \in \mathfrak{F}_{M, \delta}$ satisfies $\left(f_{1}\right)$ and $\left(f_{2}\right)$. If $f^{\prime}(t)<\lambda_{2}^{\mathrm{rad}}+1$ for every $t \in\left[0, K_{\infty}\right]$, then problem (1.1) admits only constant solutions in $H_{\mathrm{rad}}^{s}$.

Proof. We first observe that, if $M<K_{\infty}$, condition $f^{\prime}<\lambda_{2}^{\mathrm{rad}}+1$ in $\left[0, K_{\infty}\right]$ is compatible with the consequence (3.2) of $\left(f_{2}\right)$, when $\delta<\lambda_{2}^{\text {rad }}$. Let $u \in H_{\text {rad }}^{s}$ be a weak solution of (1.1). We can write $u=v+\mu$ for some $\mu \in \mathbb{R}$ and $v \in H_{\text {rad }}^{s}$ with

$$
\begin{aligned}
& \int_{\Omega} v d x=0 \text { and } \\
& \lambda_{2}^{\mathrm{rad}} \int_{\Omega} v^{2} d x \leq \frac{c_{n, s}}{2} \iint_{\mathbb{R}^{2 n} \backslash\left(\Omega^{c}\right)^{2}} \frac{|v(x)-v(y)|^{2}}{|x-y|^{n+2 s}} d x d y+\int v_{\Omega} v^{2} d x .
\end{aligned}
$$

Using the definition of weak solution for $u=v+\mu$ and testing with $v$, we get

$$
\begin{aligned}
& \left(\lambda_{2}^{\mathrm{rad}}+1\right) \int_{\Omega} v^{2} d x \leq \frac{c_{n, s}}{2} \iint_{\mathbb{R}^{2 n} \backslash\left(\Omega^{c}\right)^{2}} \frac{|v(x)-v(y)|^{2}}{|x-y|^{n+2 s}} d x d y+\int_{\Omega} v^{2} d x \\
& \quad=\int_{\Omega} f(v+\mu) v d x=\int_{\Omega}[f(v+\mu)-f(\mu)] v d x=\int_{\Omega} f^{\prime}(\mu+\omega v) v^{2} d x,
\end{aligned}
$$

where $\omega=\omega(x)$ satisfies $0 \leq \omega \leq 1$ in $\Omega$. Using that $\|u\|_{L^{\infty}(\Omega)} \leq K_{\infty}$, we deduce that $\| \mu+$ $\omega v \|_{L^{\infty}(\Omega)} \leq K_{\infty}$. Therefore, since by assumption $f^{\prime}(\mu+\omega v)<\lambda_{2}^{\mathrm{rad}}+1$, we conclude that it must be $v=0$ and thus $u$ identically constant.

Remark 5.5. Some further comments on the condition $\left(f_{3}\right)$ and its variants are now in order. In the local setting, it was first conjectured in [4] and then proved in $[3,12,5]$ that if $f^{\prime}\left(u_{0}\right)$ satisfies

$$
f^{\prime}\left(u_{0}\right)>1+\lambda_{k+1}^{\mathrm{rad}}(R) \quad \text { for some } k \geq 1,
$$

where $\lambda_{k+1}^{\mathrm{rad}}(R)$ is the $(k+1)$-st radial eigenvalue of the Neumann Laplacian in $B_{R}$, then the Neumann problem $-\Delta u+u=f(u)$ in $B_{R}$ admits a radial positive solution having exactly $k$ intersections with the constant $u_{0}$. It would be interesting to prove a similar result also in this fractional setting. It is worth stressing that the solution $u$ that we find in the present paper is morally the one with one intersection with $u_{0}$. This is due to the monotonicity of $u \in \mathcal{C}_{*}$, the identity holding for solutions of (1.1) 


$$
\int_{\Omega} u d x=\int_{\Omega} f(u) d x,
$$

and the fact that $f(t)<t$ for $t \in\left(u_{-}, u_{0}\right)$ and $f(t)>t$ in $\left(u_{0}, u_{+}\right)$, cf. (4.13) and (4.14).

We conclude this remark observing that, since $\lambda_{k}^{\mathrm{rad}}(R) \rightarrow 0$ as $R \rightarrow \infty$, condition (5.9) can be also read as a condition on the size of the domain $B_{R}$.

\section{Acknowledgments}

This work was partially supported by Gruppo Nazionale per l'Analisi Matematica, la Probabilità e le loro Applicazioni (GNAMPA) of the Istituto Nazionale di Alta Matematica (INdAM). The authors are grateful to Proff. Lorenzo Brasco and Benedetta Noris for useful discussions on the subject. The authors acknowledge the supports of both Departments of Mathematics of the Universities of Bologna and of Turin for their visits in Bologna and in Turin, during which parts of this work have been achieved. E. C. is supported by MINECO grants MTM2014-52402-C3-1-P and MTM2017-84214-C2-1-P, and is part of the Catalan research group 2014 SGR 1083. F. C. is partially supported by the INdAM/GNAMPA Project 2019 "Il modello di Born-Infeld per l'elettromagnetismo nonlineare: esistenza, regolarità e molteplicità di soluzioni" and the project of the University of Turin Ricerca Locale 2018 Linea B (CDD 09/07/2018)-“Problemi non lineari” COLF_RILO_18_01.

\section{References}

[1] N. Abatangelo, A remark on nonlocal Neumann conditions for the fractional Laplacian, preprint, arXiv:1712.00320, 2017.

[2] R.A. Adams, J.J.F. Fournier, Sobolev Spaces, second edition, Pure and Applied Mathematics (Amsterdam), vol. 140, Elsevier/Academic Press, Amsterdam, 2003.

[3] D. Bonheure, C. Grumiau, C. Troestler, Multiple radial positive solutions of semilinear elliptic problems with Neumann boundary conditions, Nonlinear Anal. 147 (2016) 236-273.

[4] D. Bonheure, B. Noris, T. Weth, Increasing radial solutions for Neumann problems without growth restrictions, Ann. Inst. Henri Poincaré, Anal. Non Linéaire 29 (2012) 573-588.

[5] A. Boscaggin, F. Colasuonno, B. Noris, Multiple positive solutions for a class of $p$-Laplacian Neumann problems without growth conditions, ESAIM Control Optim. Calc. Var. 24 (4) (2018) 1625-1644.

[6] L. Brasco, M. Squassina, Y. Yang, Global compactness results for nonlocal problems, Discrete Contin. Dyn. Syst., Ser. S 11 (3) (2018) 391-424.

[7] F. Colasuonno, A p-Laplacian Neumann problem with a possibly supercritical nonlinearity, Rend. Semin. Mat. Univ. Politec. Torino 74 (3-4) (2016) 113-122.

[8] F. Colasuonno, B. Noris, A p-Laplacian supercritical Neumann problem, Discrete Contin. Dyn. Syst. 37 (6) (2017) 3025-3057.

[9] C. Cowan, A. Moameni, A new variational principle, convexity, and supercritical Neumann problems, Trans. Am. Math. Soc. (2019).

[10] K. Deimling, Ordinary Differential Equations in Banach Spaces, Lecture Notes in Mathematics, vol. 596, SpringerVerlag, Berlin-New York, 1977.

[11] S. Dipierro, X. Ros-Oton, E. Valdinoci, Nonlocal problems with Neumann boundary conditions, Rev. Mat. Iberoam. 33 (2) (2017) 377-416.

[12] R. Ma, T. Chen, Y. Lu, On the Bonheure-Noris-Weth conjecture in the case of linearly bounded nonlinearities, Discrete Contin. Dyn. Syst., Ser. B 21 (8) (2016) 2649-2662.

[13] X. Ros-Oton, J. Serra, The Pohozaev identity for the fractional Laplacian, Arch. Ration. Mech. Anal. 213 (2) (2014) 587-628.

[14] E. Serra, P. Tilli, Monotonicity constraints and supercritical Neumann problems, Ann. Inst. Henri Poincaré, Anal. Non Linéaire 28 (1) (2011) 63-74. 
[15] W. Sickel, L. Skrzypczak, J. Vybiral, On the interplay of regularity and decay in case of radial functions I. Inhomogeneous spaces, Commun. Contemp. Math. 14 (1) (2012) 1250005.

[16] L. Silvestre, Regularity of the obstacle problem for a fractional power of the Laplace operator, Commun. Pure Appl. Math. 60 (1) (2007) 67-112.

[17] H. Triebel, Theory of Function Spaces. Modern Birkhäuser Classics, Birkhäuser/Springer Basel AG, Basel, 2010, Reprint of 1983 edition, also published in 1983 by Birkhäuser Verlag. 